\title{
Overexpression of a Novel ERF-X-Type Transcription Factor, OsERF106MZ, Reduces Shoot Growth and Tolerance to Salinity Stress in Rice
}

\author{
Hung-Chi Chen ${ }^{1+}{ }^{\oplus}$, Tzu-Cheng Chien ${ }^{1 \dagger}$, Tsung-Yang Chen ${ }^{1}$, Ming-Hau Chiang ${ }^{1}$, Ming-Hsin Lai ${ }^{2}$ and \\ Men-Chi Chang ${ }^{1 *}$
}

\begin{abstract}
Transcription factors (TFs) such as ethylene-responsive factors (ERFs) are important for regulating plant growth, development, and responses to abiotic stress. Notably, more than half of the rice ERF-X group members, including ethylene-responsive factor 106 (OsERF106), are abiotic stress-responsive genes. However, their regulatory roles in abiotic stress responses remain poorly understood. OsERF106, a salinity-induced gene of unknown function, is annotated differently in RAP-DB and MSU RGAP. In this study, we isolated a novel (i.e., previously unannotated) OsERF106 gene, designated OsERF106MZ (GenBank accession No. MZ561461), and investigated its role in regulating growth and the response to salinity stress in rice. OsERF106MZ is expressed in germinating seeds, primary roots, and developing flowers. Overexpression of OSERF106MZ led to retardation of growth, relatively high levels of both malondialdehyde (MDA) and reactive oxygen species (ROS), reduced catalase (CAT) activity, and overaccumulation of both sodium $\left(\mathrm{Na}^{+}\right)$and potassium $\left(\mathrm{K}^{+}\right)$ions in transgenic rice shoots. Additionally, the expression of OsHKT1.3 was downregulated in the shoots of transgenic seedlings grown under both normal and $\mathrm{NaCl}$-treated conditions, while the expression of OsAKT1 was upregulated in the same tissues grown under $\mathrm{NaCl}$-treated conditions. Further microarray and $\mathrm{PPCR}$ analyses indicated that the expression of several abiotic stress-responsive genes such as OSABI5 and OsSRO1C was also altered in the shoots of transgenic rice grown under either normal or $\mathrm{NaCl}$-treated conditions. The novel transcription factor OsERF106MZ negatively regulates shoot growth and salinity tolerance in rice through the disruption of ion homeostasis and modulation of stress-responsive gene expression.
\end{abstract}

Keywords: AP2/ERF, lon homeostasis, Salinity stress, Shoot growth, Transcription factor

\section{Background}

Soil salinity is one of the major constraints limiting crop growth and production. In general, soil with an electrical conductivity of saturated soil-paste extract (ECe) of $4 \mathrm{dS}$ $\mathrm{m}^{-1}$ (equivalent to $40 \mathrm{mM} \mathrm{NaCl}$ ) or more is considered to

\footnotetext{
*Correspondence: menchi@ntu.edu.tw

†Hung-Chi Chen and Tzu-Cheng Chien have contributed equally to this work

1 Department of Agronomy, National Taiwan University, No. 1, Section 4, Roosevelt Road, Taipei, Taiwan, ROC

Full list of author information is available at the end of the article
}

be saline (United State Department of Agriculture 2016). It is speculated that more than $20 \%$ of all cultivated land and almost half of all irrigated land face the problem of soil salinization (FAO, http://www.fao.org/home/en/). In plants, excessive salt uptake from the soil can lead to an imbalance of cellular ions, resulting in osmotic dehydration of the cells and ion toxicity. However, plants have evolved a sophisticated regulatory mechanism to deal with salinity stress. The osmotic signal can be transduced into the nucleus via both abscisic acid (ABA)dependent (e.g., involving calcium-dependent protein 
kinases [CDPKs] and transcription factors [TFs] such as myelocytomatosis/myeloblastosis [MYC/MYB]) and ABA-independent (e.g., via C-repeat binding factor/ dehydration-responsive element binding protein $[\mathrm{CBF} /$ DREB] TFs) pathways, which in turn activate the expression of downstream stress-responsive genes that confer tolerance to salinity stress in plants (for a review, see Kumar et al. 2013). Additionally, the regulation of sodium uptake, transport, and compartmentation is also important for plant survival under salinity stress. Several types of transporters, such as HKTs (high-affinity $\mathrm{K}^{+}$transports), NHXs ( $\mathrm{Na}^{+} / \mathrm{H}^{+}$exchangers), and SOS (salt overly sensitive), have been shown to be involved in adjusting sodium homeostasis (Deinlein et al. 2014). When plants encounter salinity stress, these transporters act collectively to reduce the accumulation of cytosolic sodium, thereby alleviating sodium toxicity in cells (for a review, see Isayenkov and Maathuis 2019). Therefore, both TFs and the ion transporters function as key components that integrate signal transduction, stress-responsive gene expression, and sodium/potassium homeostasis to enhance salinity tolerance in plants.

TFs are proteins that regulate the transcription of target genes by directly binding to a specialized sequence known as a cis-regulatory (or cis-acting) element (CRE), in the promoter regions. Therefore, it can be expected that almost all TFs contain at least one DNA-binding domain (DBD) that recognizes and attaches to a specific CRE in the promoter region of target genes (Mitchell and Tjian 1989; Ptashne and Gann 1997). To date, at least 2296, 3308, 1891, and 2048 TFs have been found in Arabidopsis (Arabidopsis thaliana), maize (Zea mays), indica rice (Oryza sativa subsp. indica), and japonica rice (Oryza sativa subsp. japonica), respectively (Additional file 1: Figure S1). Notably, more than $45 \%$ of TFs in Arabidopsis, maize, and rice belong to the bHLH (basic helix-loop-helix), bZIP (basic leucine zipper), AP2/ERF (APETALA2/ethylene-responsive factor), C2H2 (Cys2/ His2-type zinc finger protein), MYB, NAC (NAM, ATAF, and CUC), and WRKY families (Additional file 1: Figure S1), which implies that members of these seven families are extensively involved in the regulation of physiological processes in plants, including abiotic stress responses. For example, AtbHLH112 increases the expression of $P O D$ (peroxidase) and SOD (superoxide dismutase) genes to improve ROS scavenging ability, which confers tolerance to drought and salinity stresses in Arabidopsis (Liu et al. 2015). In rice, NAC022 plays a positive role in drought and salinity tolerance through the increased expression of two ABA biosynthetic genes (OsNCED3 [nine-cis-epoxycarotenoid dioxygenase 3] and OsPSY [phytoene synthase]), signaling and regulatory genes (such as OsDREB2a), and late stress-responsive genes (such as OsLEA3 [late embryogenesis abundant 3]) (Hong et al. 2016). Therefore, these previous studies illustrated that TF-regulated gene expression plays a pivotal role in the management of abiotic stresses among various plants.

AP2/ERFs comprise the second largest TF family in Arabidopsis, maize, and japonica rice and the largest TF family in indica rice. To date, at least 176, 261, 170, and 189 AP2/ERFs have been identified in Arabidopsis, maize, indica rice, and japonica rice, respectively (Additional file 1: Figure S1). AP2/ERFs are characterized by a conserved DBD known as the AP2/EREBP (APETALA2/ ethylene-responsive element binding protein) domain, which consists of approximately 60 amino acid (aa) residues (Ohme-Takagi and Shinshi 1995). Based on the similarity and number of AP2/EREBP domains as well as the occurrence of additional domains, the AP2/ERFs can be broadly categorized into four major subfamilies: (1) the AP2 subfamily; (2) the RAV (related to ABA insensitive 3/viviparous 1 ) subfamily; (3) the CBF/DREB subfamily; and (4) the ERF subfamily, containing one AP2/EREBP domain with a conserved alanine residue at position 14 and a conserved aspartic acid at position 19 (Sakuma et al. 2002; Nakano et al. 2006). Based additionally on gene structure (e.g., exon-intron organization), the CBF/ DREB subfamily can be further classified into 4 distinct groups (I-IV) in Arabidopsis and japonica rice, while the ERF subfamily can be further divided into 8 groups (V-X, VI-like, and Xb-like) in Arabidopsis and 11 groups (V-XIV and VI-like) in japonica rice (Nakano et al. 2006). In addition, CBF/DREB proteins regulate the transcription of target genes by binding to a CRE known as the CRT/DRE (C-repeat/dehydration-responsive element [5'TACCGACAT-3']) box in their promoters, but the ERF binds to the GCC (5'-AGCCGCC-3') box (Ohme-Takagi and Shinshi 1995; Jiang et al. 1996). In Arabidopsis, several CBF/DREB-encoding genes have been extensively characterized and shown to be involved in abiotic stress responses. For example, CBF/DREB-IIIc-type TFs together with another cold-responsive TF, AtbHLH116/ ICE1 (inducer of CBF expression 1), directly activate a majority of CRT/DRE-containing CORs (cold responsive genes), which contributes positively to cold tolerance (Chinnusamy et al. 2003; Zhao et al. 2016; Liu et al. 2018). Interestingly, AtERF006/RAP2.1 (related to AP2 1 [At6g46768]), a cold-induced CBF/DREB-IIa-type TF, acts as a repressor of CBF/DREB1-mediated cold acclimation, which negatively regulates cold tolerance (Dong and Liu 2010). These studies suggest that AP2/ERFs can antagonize each other to adjust plant growth in response to abiotic stresses.

Rice is one of the most important food crops in the world. However, rice is highly sensitive to salinity stress, especially at the young seedling stage. The threshold ECe 
for most cultivated rice varieties is $3 \mathrm{ds} / \mathrm{m}^{-1}$, and each $1 \%$ increase in ECe beyond this threshold can cause an approximately $12 \%$ decline in the yield of rice (Maas and Hoffman 1997). Therefore, efforts to decipher the regulatory mechanisms of salinity tolerance are essential for the development of salt-tolerant rice varieties. To identify which uncharacterized OsERFs may be involved in the salinity stress response, we analyzed the expression profiles of OsERFs from publicly available microarray datasets (GSE6901 and GSE14275), and several salinity stress-responsive OsERFs with an unknown function were found. Among these genes, the expression of ethylene-responsive factor 106 (OsERF106 [LOC Os08g42550]) was upregulated under salinity stress conditions. OsERF106 is a member of the rice ERF-Xc subgroup along with OsERF105 (LOC_Os05g36100) and OsERF107 (LOC_Os02g32140) (Nakano et al. 2006). The functions of rice ERF-Xc subgroup genes have not yet been characterized. Moreover, the annotation of putative OsERF106 transcripts in the Rice Annotation Project Database (RAP-DB, http://rapdb.dna.affrc.go. $\mathrm{jp} /$ ) are inconsistent with those in the Michigan State University Rice Genome Annotation Project Database (MSU RGAP, http://rice.plantbiology.msu.edu/). In this study, we isolated a cDNA encoding a previously unannotated OsERF106 and characterized its role in the rice plant response to salinity stress through a reverse genetic approach.

\section{Results}

\section{Molecular Cloning and Structural Characterization} of a Salinity Stress-Responsive TF Gene, OsERF106MZ

In silico analyses of microarray-based gene expression profiles indicated that the expression of OsERF106 is upregulated under salinity stress but is undetectable under cold, drought, or heat stress (Fig. 1a). Notably, the annotation of putative OsERF106-encoding transcripts in the RAP-DB was different from that in the MSU RGAP. Two transcript variants of OsERF106 (Os08t053790001 and Os08t0537900-02) were documented in the RAP-DB, while six alternative splice forms of OsERF106 (LOC_Os08g42550.1 to LOC_Os08g42550.6) were described in the MSU RGAP (Additional file 1: Figure S2). Among these putative OsERF106-encoding transcripts, Os08t0537900-01 (as well as LOC_Os08g42550.2 to LOC_Os08g42550.6) encodes an O-fucosyltransferase (O-FucT) of 453 aa in length, but this enzyme does not contain an AP2/EREBP domain, a defining feature of the AP2/ERF proteins (Additional file 1: Figures S2 and S3). The coding sequence (CDS) of Os08t053790002 seems to be incorrect because it lacks an ATG-start codon and encodes an unknown protein without an AP2/EREBP domain (Additional file 1: Figures S2 and
S4). LOC_Os08g42550.1 encodes a 541-aa protein that contains an O-FucT-like domain and an AP2/EREBP domain. However, the LOC_Os08g42550.1-encoded protein is homologous to AtO-FucT13 and GmO-FucT13 but is not homologous to any AtAP2/ERF or GmAP2/ ERF (Additional file 1: Figures S2 and S5). This is unusual because no AP2/EREBP domain-containing O-FucTlike proteins have been identified to date. Furthermore, we were not able to amplify the full-length CDS of LOC_Os08g42550.1 by RT-PCR (reverse transcriptionPCR). Therefore, we performed rapid amplification of cDNA ends-PCR (RACE-PCR) using gene-specific primers (GSPs) to clarify the CDS of OsERF106. The PCRamplified product was approximately $1.3 \mathrm{~kb}$ in length, including a 5' untranslated region (5' UTR) of $351 \mathrm{bp}$, an open reading frame (ORF) of 648 bp encoding a previously unannotated OsERF106, and a 3' UTR of $333 \mathrm{bp}$. This previously unannotated OsERF106 gene, designated OsERF106MZ (GenBank accession No. MZ561461), consisted of two exons, and the encoded protein contained a typical AP2/EREBP domain spanning residues 36 to 93 (Additional file 1: Figure S6). Phylogenetic analysis revealed that OsERF106MZ (together with OsERF105 [LOC_Os05g36100] and OsERF107 [LOC_Os02g32140]) is homologous to AtERF108/RAP2.6 (At1g43160) and AtERF113/RAP2.6L (At5g13330) but is not highly homologous to AtO-FucT13 or GmO-FucT13 (Additional file 1: Figure S7).

To verify whether the expression of OsERF106MZ was upregulated under salinity stress, histochemical staining was performed in OsERF106MZp:: $\beta$-glucuronidase (GUS) transgenic rice. Under normal $(0 \mathrm{mM} \mathrm{NaCl})$ conditions, GUS was rarely expressed in the shoots of 8-day-old transgenic seedlings; however, its expression was obviously enhanced in response to $150 \mathrm{mM}$ $\mathrm{NaCl}$ (Fig. 1b). Additionally, the expression pattern of OsERF106MZ was similar to that of OsSOS1 (LOC_ Os12G44360), a well-known salinity stress-responsive gene that was upregulated under $150 \mathrm{mM} \mathrm{NaCl}$ conditions in Tainung 67 (Oryza sativa L. spp. japonica cv. Tainung 67) and Hitomebore (Oryza sativa L. spp. japonica cv. Hitomebore) seedlings (Fig. 1c). These data indicate that OsERF106MZ is a salinity stress-responsive TF gene.

\section{Spatiotemporal OsERF106MZ Expression and OsERF106MZ Localization}

The analysis of spatiotemporal gene expression trajectories and protein localization may offer valuable insights into gene function. Thus, we examined the spatiotemporal expression pattern of OsERF106MZ by detecting GUS signals in OsERF106MZp::GUS transgenic plants from the beginning of seed germination to the reproductive stage. After imbibition, the GUS signal was localized to 


\section{(A)}

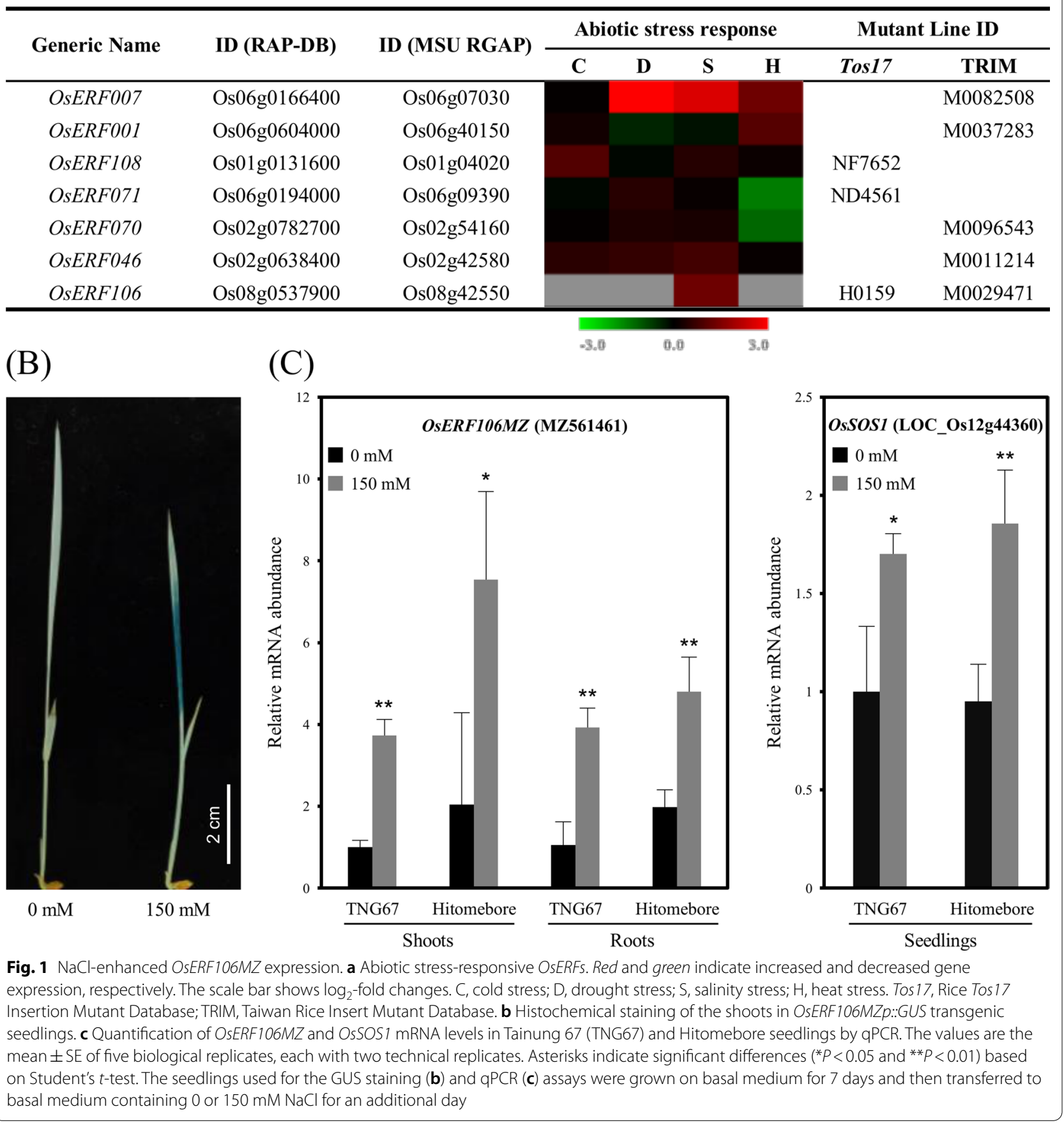

the embryo of germinating OsERF106MZp::GUS transgenic seeds (Fig. 2a). After germination, GUS was predominantly expressed in the terrestrial tissues, such as the primary and seminal roots, of transgenic seedlings (Fig. 2a). Additionally, GUS signals were also observed in the lemma nerves, anthers, and stigmas during reproductive growth (Fig. 2b-d).
To examine the subcellular localization of OsERF106MZ, we transformed OsERF106MZ-green fluorescent protein (GFP) and GFP-OsERF106MZ constructs driven by the cauliflower mosaic virus (CaMV) $35 \mathrm{~S}$ promoter into Oncidium 'Sweet Sugar' suspension cells using electroporation. Similar to the results obtained for GFP alone, both the OsERF106MZ-GFP and 

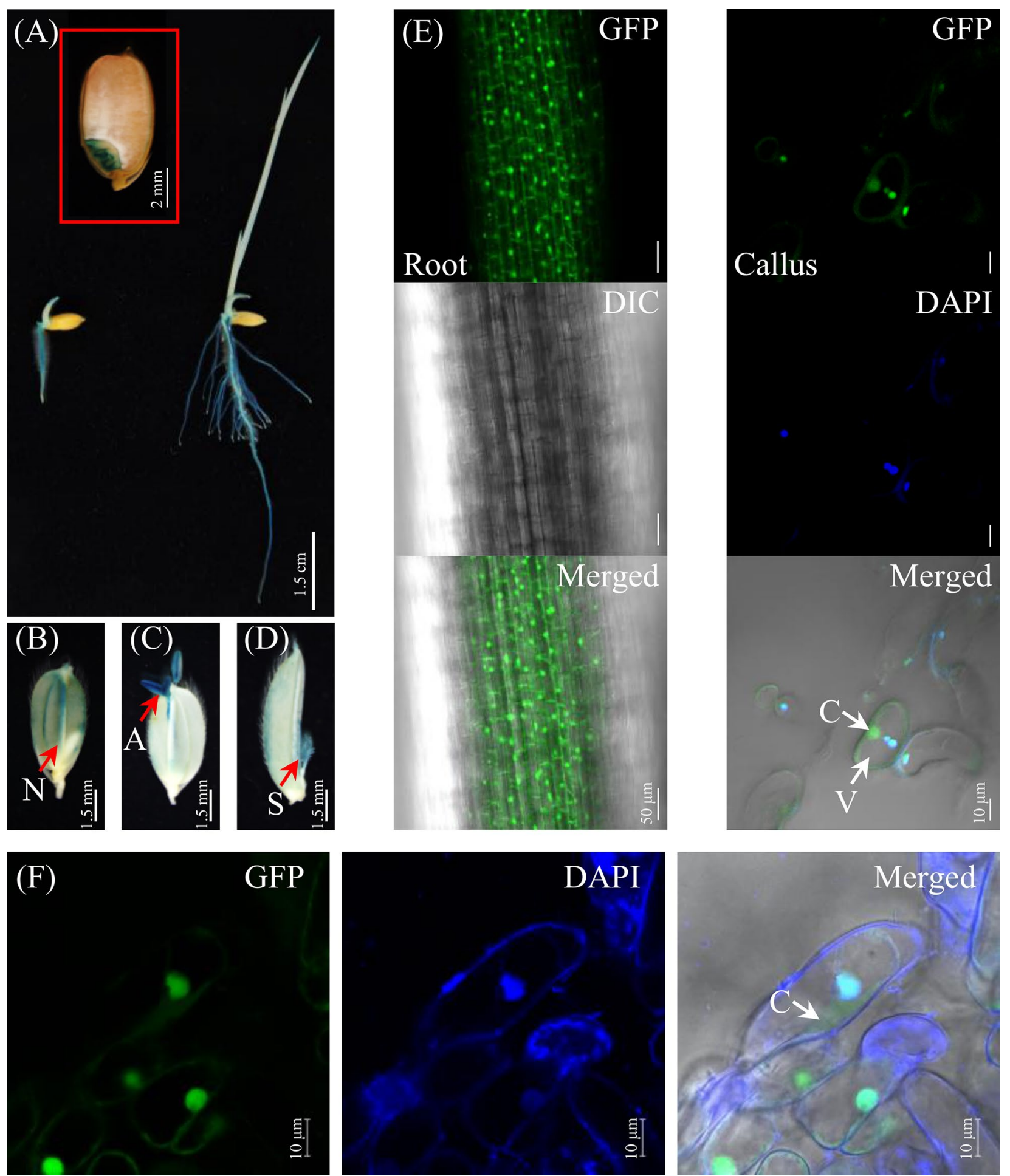

Fig. 2 The spatiotemporal expression of OsERF106MZ and the subcellular localization of OsERF106MZ-GFP in stable rice transformants. a GUS staining of germinating seeds (insert) and post-germination-stage seedlings from an OsERF106MZp::GUS transformant. b-d GUS activity in developing flowers of an OsERF106MZp::GUS transformant. N, nerve; A, anther; S, stigma. e The presence of OsERF106MZ-GFP in the root vasculature (left panels) and callus cells (right panels). f Subcellular localization of OsERF106MZ-GFP in the NaCl-treated callus cells. The calli induced from the husk-removed seeds harboring 35Sp::OSERF106MZ-GFP were treated with $150 \mathrm{mM} \mathrm{NaCl}$ for $24 \mathrm{~h}$ and then subjected to GFP visualization by confocal microscopy. C, cytosol; $V$, vacuole 
GFP-OsERF106MZ fusion proteins were ubiquitously distributed throughout the entire cell (Additional file 1: Figure S8). To further verify the subcellular localization of OsERF106MZ, a 35Sp::OsERF106MZ-GFP construct was transformed into japonica rice cultivar Tainung 67 plants via stable Agrobacterium-mediated transformation. As shown in Fig. 2e, the OsERF106MZ-GFP fusion proteins were distributed throughout the cells of the root vasculature in transgenic rice seedlings. Indeed, the OsERF106MZ-GFP fusion protein not only colocalized with the nuclear 4',6-diamidino-2-phenylindole (DAPI) stain but also was present in the cytosol of non- $\mathrm{NaCl}-$ treated callus cells or $\mathrm{NaCl}$-treated callus cells (Fig. 2e, f). Therefore, these data reveal that OsERF106MZ, a salinity stress-responsive TF, does not specifically localize to the nucleus in rice cells.

\section{Overexpression of OsERF106MZ Reduces Plant Growth and Tolerance to Salinity Stress in Transgenic Rice Seedlings}

To investigate the functional role of OsERF106MZ in regulating rice growth and development, a 35Sp::OsERF106MZ construct was transformed into plants of the japonica rice cultivar Tainung 67, and homozygous OsERF106MZ-overexpressing transgenic rice plants were obtained in the $\mathrm{T}_{2}$ generation. Additionally, we searched the Rice Tos 17 Insertion Mutant Database and selected a retrotransposon insertion Oserf106 mutant line, H0159. Based on its annotation, the retrotransposon is inserted into an intron of OsERF106MZ, which is equivalent to intron 7 of LOC_Os08g42550.1 (Additional file 1: Figure S9A). Homozygous H0159 lines were identified from the segregating population by genomic DNA genotyping PCR (Additional file 1: Figure S9B). Quantitative PCR (qPCR) analysis using the primer pair OsERF106-qF and OsERF106-qR, amplifying a $108 \mathrm{bp}$ fragment downstream of the region encoding the AP2/EREBP domain, revealed that the mRNA level of OsERF106MZ was higher in transgenic rice plants harboring either 35Sp::OsERF106MZ (OE1) or 35Sp::OsERF106MZ-GFP (OE2) than in the corresponding wild-type (WT) Tainung 67 plants, while the mRNA level of OsERF106MZ was lower in the homozygous H0159 mutant than in the corresponding WT Hitomebore plant (Fig. 3a, Additional file 1: Figure S9A). Thus, the homozygous $\mathrm{H} 0159$ plant was used as an Oserf $106 \mathrm{mz}$ mutant in this study. Incidentally, no significant difference in the mRNA levels of LOC_Os08g42550.s was observed between the Oserf106mz mutant and its corresponding WT Hitomebore plant when the universal primer pair $O-F u c T$-qF and $O-F u c T$-qR was employed to amplify a common fragment of $72 \mathrm{bp}$ containing a sequence encoding part of O-FucT from the LOC_ Os08g42550.s transcript (Additional file 1: Figure S9A and $C$ ).

Because the expression of OsERF106MZ is induced by salinity stress, we subsequently examined the differences in growth performance among the OsERF106MZ-overexpressing transformants (OE1 and OE2), the Oserf106mz mutant, and the corresponding WT cultivars under both normal and $\mathrm{NaCl}$-treated conditions. As shown in Fig. $3 \mathrm{~b}$ and $\mathrm{c}$, the plant height of OsERF106MZ-overexpressing transgenic seedlings was significantly shorter than that of the corresponding WT Tainung 67 seedlings under either normal or $\mathrm{NaCl}$-treated conditions, while the root length of OsERF106MZ-overexpressing transgenic seedlings was not different from that of WT Tainung 67 seedlings. In addition to the dwarf phenotype, OsERF106MZ-overexpressing transgenic seedlings also had a relatively low rate of survival under $\mathrm{NaCl}$-treated conditions (Fig. 3d). Notably, no apparent difference in plant growth or morphology was observed between the Oserf106mz mutant and its corresponding WT Hitomebore seedlings under normal or NaCl-treated conditions (Fig. $3 \mathrm{~b}$ and c).

Phospholipids and membrane proteins are the major components of biological membranes in all living organisms. Under abiotic stress conditions, excessive lipid peroxidation can alter the composition or fluidity

\footnotetext{
(See figure on next page.)

Fig. 3 A comparison of growth patterns and salinity stress responses among OsERF106MZ-overexpressing transformants (OE1 and OE2), the Oserf106 mz mutant, and the corresponding WT cultivars under normal $(0 \mathrm{mM} \mathrm{NaCl})$ and NaCl-treated (150 mM NaCl) conditions. a Quantification of OsERF106MZ mRNA levels in OsERF106MZ-overexpressing transformants, the Oserf106mz mutant, and the corresponding WT cultivars by qPCR. The positions of the primers are indicated by purple arrows in Additional file 1: Figure S9A. The primer sequences are listed in Additional file 2: Table S1. The seedlings were grown on basal medium for 11 days and then subjected to qPCR assays. b Morphological appearance of the OsERF106MZ-overexpressing transformants, Oserf106mz mutant, and the corresponding WT cultivars. c The shoot and root lengths of the OSERF106MZ-overexpressing transformants, Oserf106mz mutant, and the corresponding WT cultivars. d Morphological phenotype and survival rate of the OsERF106MZ-overexpressing transformants and its corresponding WT cultivars. The seedlings used for the assessment of morphological characteristics such as shoot and root lengths ( $\mathbf{b}$ and $\mathbf{c}$ ) and the calculation of survival rates (d) were grown on basal medium for 7 days and then transferred to basal medium containing 0 or $150 \mathrm{mM} \mathrm{NaCl}$ for an additional 4 and 6 days, respectively. The values of shoot and root lengths (c) are the mean \pm SE of at least five biological replicates, each with two technical replicates. All survival rates (d) are the mean \pm SE of 4 independent biological replicates. Each biological replicate contains at least 20 seedlings. Asterisks indicate significant differences $\left({ }^{*} P<0.05\right.$ and $\left.{ }^{* *} P<0.01\right)$ in comparison to the corresponding WT based on Student's $t$-test
} 

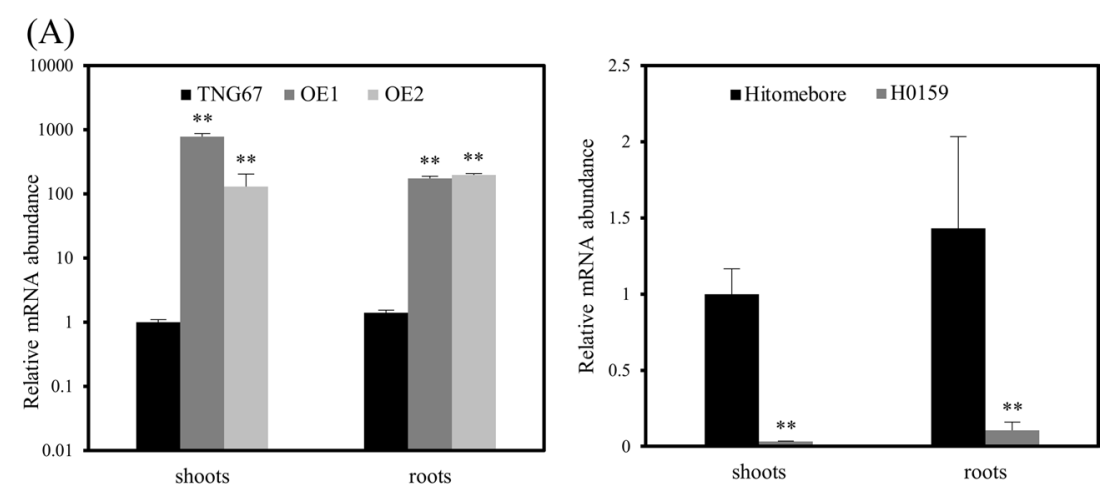

(B)
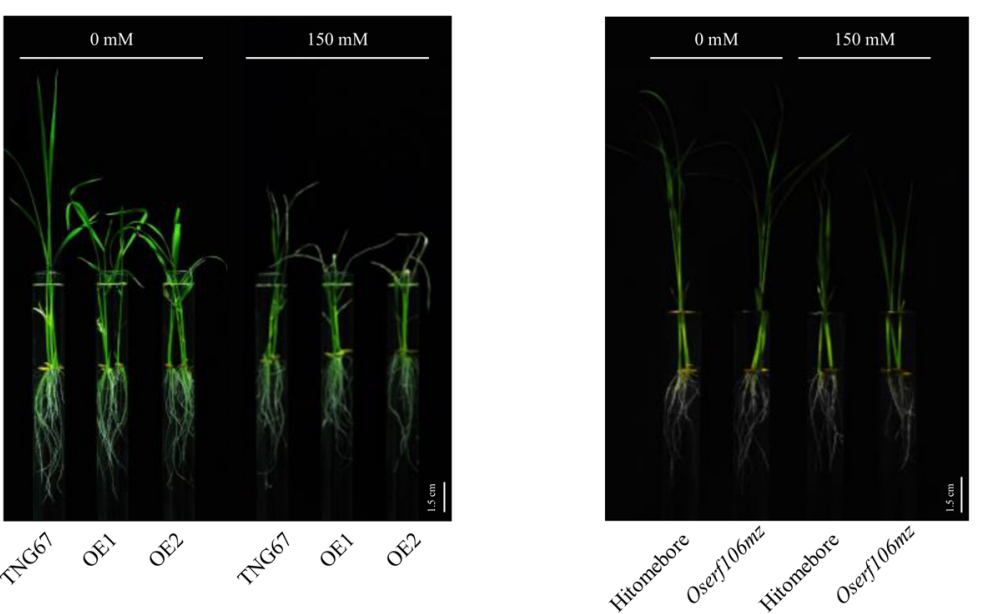

(C)
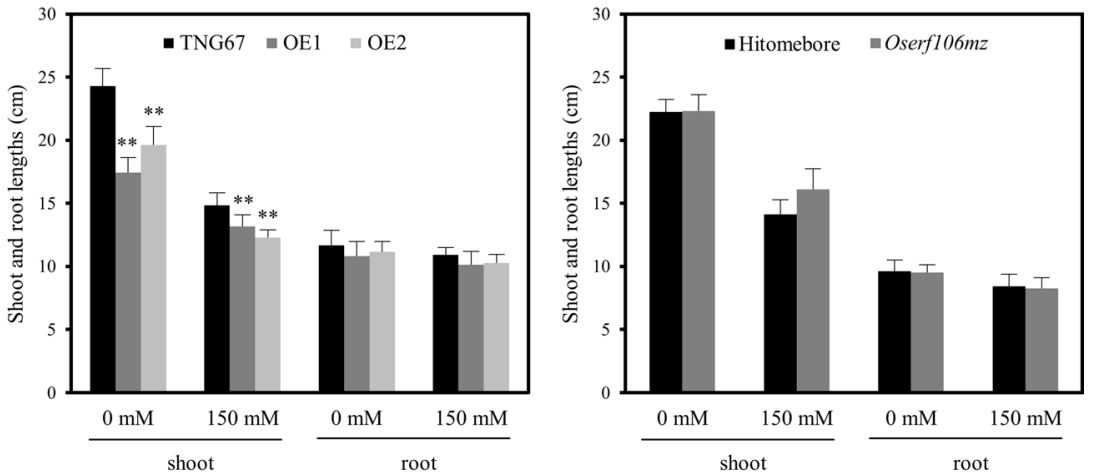

(D)
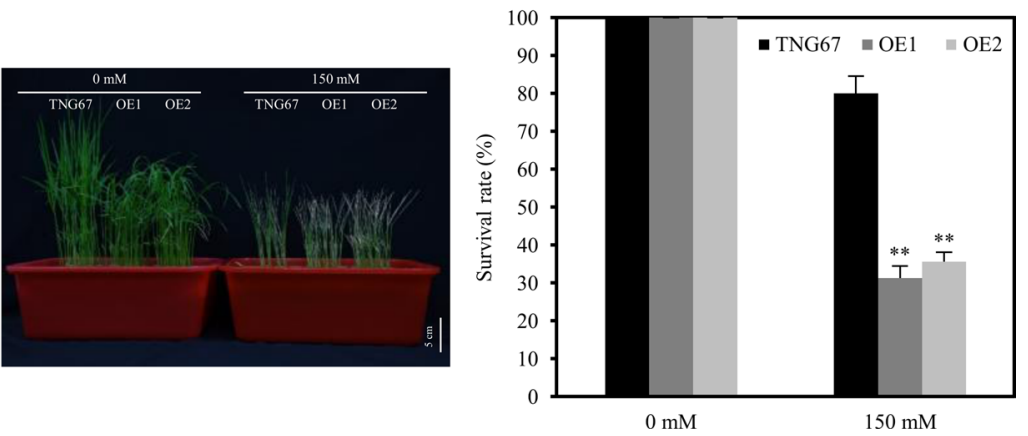

Fig. 3 (See legend on previous page.) 


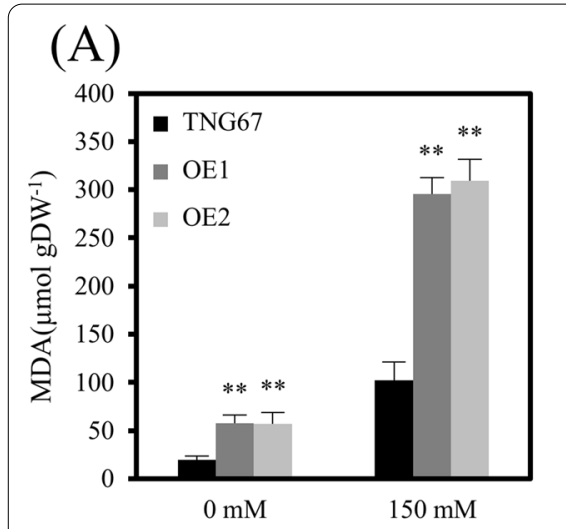

(B)
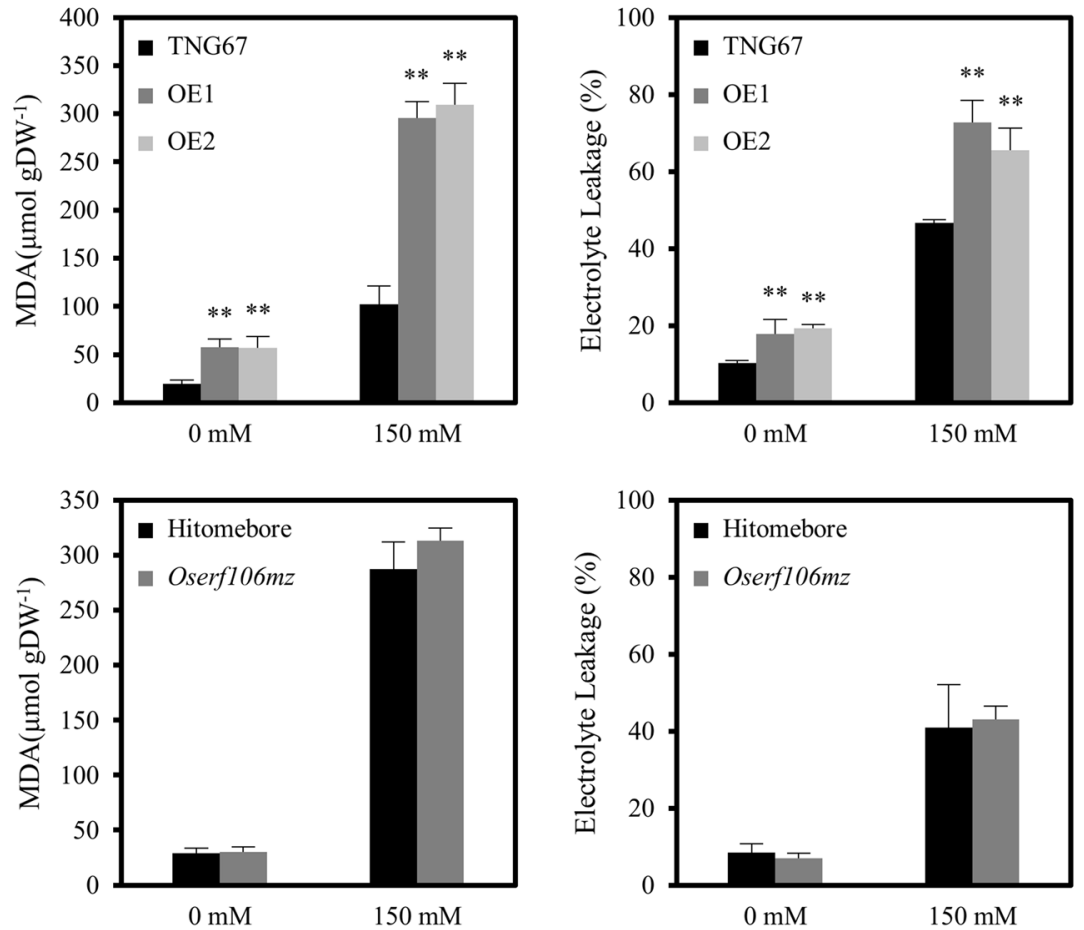

(C)

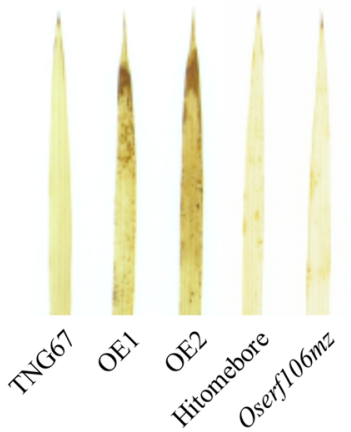

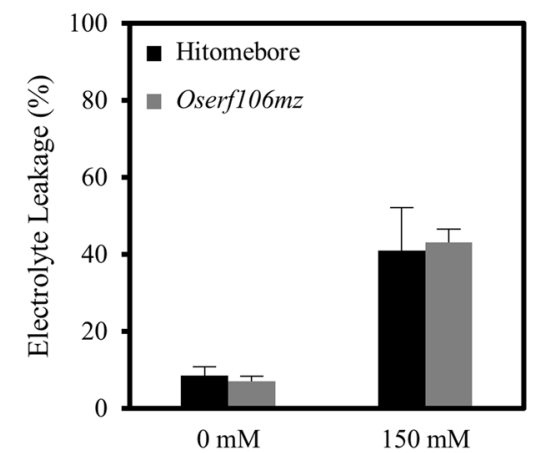

(D)
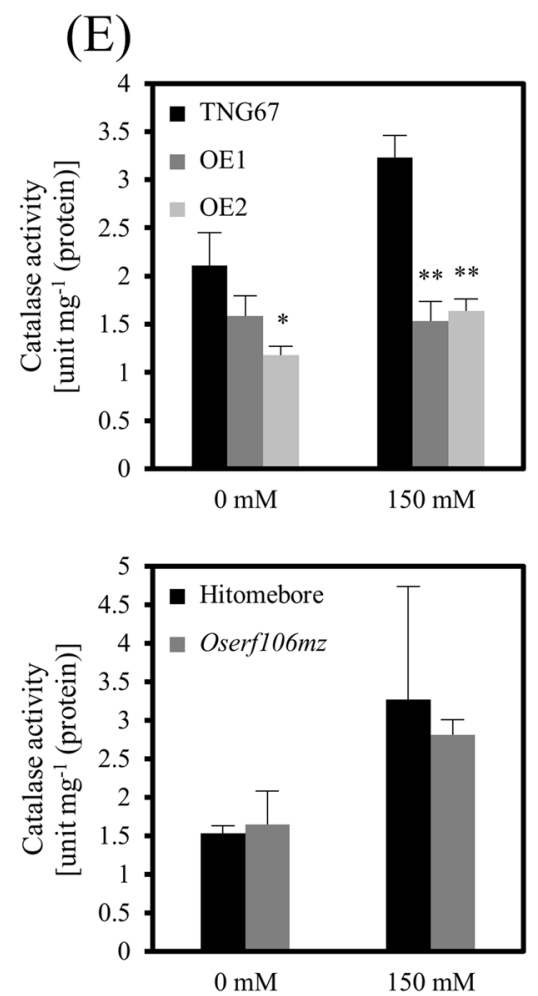

Fig. 4 Analysis of MDA content, electrolyte leakage, ROS levels, and CAT activity in the shoots of the OsERF106MZ-overexpressing transformants, Oserf106mz mutant, and the corresponding WT cultivars under normal and NaCl-treated conditions. a MDA content. b Electrolyte leakage. c DAB staining. $\mathbf{d}$ NBT staining. e CAT activity. The values are the mean \pm SE of five biological replicates, each with two technical replicates. Asterisks indicate significant differences $\left({ }^{*} P<0.05\right.$ and $\left.{ }^{* *} P<0.01\right)$ in comparison to the corresponding WT based on Student's $t$-test. For these assays, seedlings were grown on basal medium for 7 days and then transferred to basal medium containing 0 or $150 \mathrm{mM} \mathrm{NaCl}$ for an additional day

of membranes, further leading to membrane damage. Therefore, we examined the malondialdehyde (MDA, an index of lipid peroxidation) and relative electrolyte leakage (an index reflecting the changes of cell membrane permeability) levels among the OsERF106MZoverexpressing transformants, the Oserf106mz mutant, and the corresponding WT cultivars. Under both normal and $\mathrm{NaCl}$-treated conditions, the MDA content and electrolyte leakage in the shoots of OsERF106MZoverexpressing transformants were significantly higher than those of the corresponding WT Tainung 67 plants (Fig. 4a and b). The relatively high level of MDA was also observed in the roots of OsERF106MZ-overexpressing transformants under normal conditions (Additional file 1: Figure S10A). Additionally, histochemical analyses with 3,3'-diaminobenzidine (DAB) and nitro blue tetrazolium (NBT) showed that the ROS levels in the leaves of OsERF106MZ-overexpressing transformants were higher than those in the WT Tainung 67 plants under either normal or $\mathrm{NaCl}$-treated conditions 


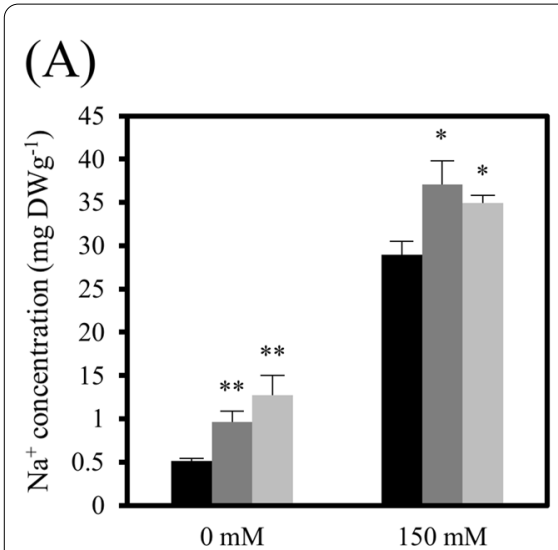

\section{- TNG67 $\quad$ OE1 $\square$ OE2}
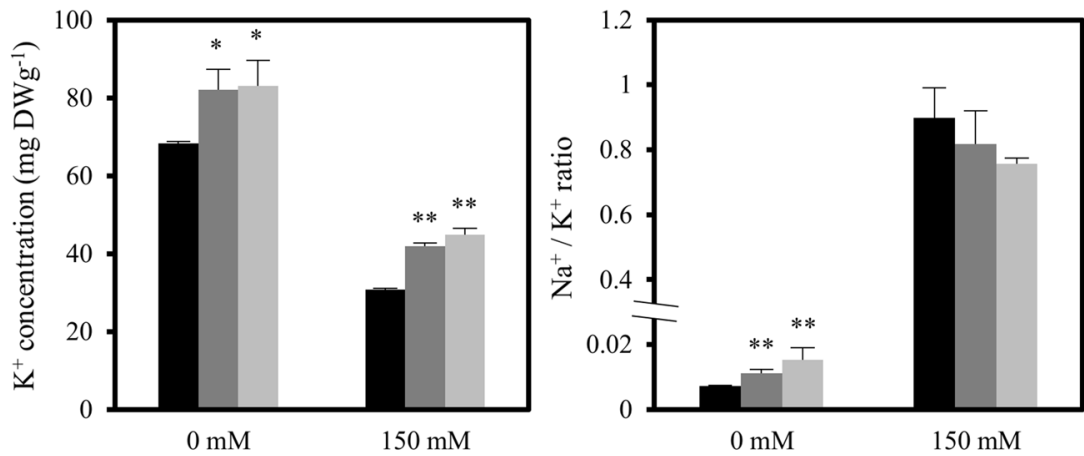

(B)
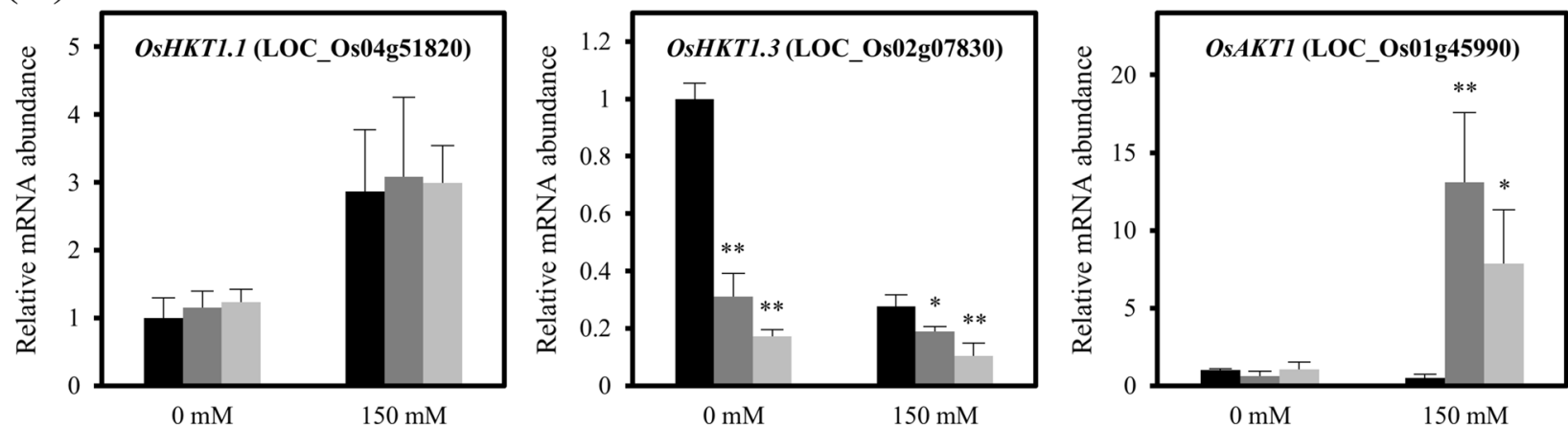

Fig. 5 Overexpression of OSERF 106MZ impairs the homeostasis of sodium and potassium in transgenic shoots. a Na ${ }^{+}$and $\mathrm{K}^{+}$contents and $\mathrm{Na}^{+} /$ $\mathrm{K}^{+}$ratio in the shoots of Tainung 67 and OsERF106MZ-overexpressing rice plants. b Quantification of OsHKT1.1, OsHKT1.3, and OsAKT1 mRNAs in the shoots of Tainung 67- and OSERF106MZ-overexpressing rice plants. The values are the mean \pm SE of at least four biological replicates, each with two technical replicates. Asterisks indicate significant differences $\left({ }^{*} P<0.05\right.$ and $\left.{ }^{*} P<0.01\right)$ in comparison to the corresponding WT (Tainung 67) based on Student's t-test. The seedlings were grown on basal medium for 7 days and then transferred to basal medium containing 0 or $150 \mathrm{mM} \mathrm{NaCl}$ for an additional 4 days

(Fig. 4c and d). In contrast, CAT activity was significantly decreased in the shoots of OsERF106MZ-overexpressing transformants compared to that in the WT Tainung 67 plants under either normal or NaCl-treated conditions. Furthermore, $\mathrm{NaCl}$-induced CAT activity was almost completely abolished in the shoots of OsERF106MZ-overexpressing transformants (Fig. 4e). Notably, no significant difference in ROS levels or CAT activity was observed between the Oserf106mz mutant and the corresponding WT Hitomebore seedlings under either normal or NaCl-treated conditions, which was similar to the morphological assessment results (Fig. 3 vs. Figure 4). Taken together, these data reveal that overexpression of OsERF106MZ in rice negatively regulates shoot growth and salinity stress tolerance, but its function in regulating these physiological processes may be redundant with those of other homologs.

\section{Overexpression of OsERF106MZ Leads to Excessive Accumulation of $\mathrm{Na}^{+}$in Transgenic Rice Seedlings}

To understand the nature of the relationship between plant growth and salinity stress responses in OsERF106MZ-overexpressing transgenic rice seedlings, we further examined the differences in $\mathrm{Na}^{+}$and $\mathrm{K}^{+}$levels between Tainung 67 and OsERF106MZ-overexpressing rice plants by using inductively coupled plasma-optical emission spectrometry (ICP-OES). Under normal conditions, $\mathrm{Na}^{+}$levels were increased by at least $85 \%$ and $\mathrm{K}^{+}$ levels were increased by approximately $20 \%$ in both OE 1 and OE2 shoots when normalized to the levels in the corresponding Tainung 67 shoots (Fig. 5a). These changes led to a higher $\mathrm{Na}^{+} / \mathrm{K}^{+}$ratio in OsERF106MZ-overexpressing shoots grown under normal conditions. Similarly, the levels of $\mathrm{Na}^{+}$and $\mathrm{K}^{+}$were also higher in both OE1 and OE2 shoots than in the corresponding Tainung 
67 shoots under $\mathrm{NaCl}$-treated conditions. Moreover, the relatively high level of $\mathrm{Na}^{+}$was also observed in the roots of OE plants under normal conditions (Additional file 1: Figure S10B). These data imply that OsERF106MZ may be involved in regulating sodium and potassium homeostasis. Accordingly, we examined the changes in the expression levels of several ion transport-related genes in OsERF106MZ-overexpressing shoots grown under normal or $\mathrm{NaCl}$-treated conditions. As shown in Fig. 5b, no obvious differences in the expression of OsHKT1.1 were found between the Tainung 67- and OsERF106MZoverexpressing rice shoots under either normal or $\mathrm{NaCl}$ treated conditions. However, the expression levels of OsHKT1.3 and OsAKT1 (a rice gene homologous to Arabidopsis $\mathrm{K}^{+}$transporter 1) were significantly higher and lower, respectively, in OsERF106MZ-overexpressing rice shoots than in Tainung 67 shoots under normal and/ or $\mathrm{NaCl}$-treated conditions. Taken together, these results reveal that the overexpression of OsERF106MZ interferes with ion transport and subsequently causes the excessive accumulation of $\mathrm{Na}^{+}$and $\mathrm{K}^{+}$in the shoots, which may exacerbate ion toxicity, resulting in shoot growth retardation of the transgenic rice seedlings.

\section{Transcriptomic Analysis of OsERF106MZ-Overexpressing Transgenic Rice Shoots}

To gain better insight into the molecular mechanisms underlying OsERF106MZ-mediated responses to salinity stress in rice shoots, we performed a comparative transcriptomic analysis of Tainung 67- and OsERF106MZ-overexpressing shoots under both normal and $\mathrm{NaCl}$-treated conditions. After background correction and normalization, a total of 1746 and 1553 differentially expressed genes (DEGs) were identified from the shoots of OsERF106MZ-overexpressing transgenic rice seedlings grown under normal and $\mathrm{NaCl}$-treated conditions, respectively (Fig. 6, Additional file 3: Data S1). Among these genes, 784 and 846 DEGs were upregulated in the shoots of OsERF106MZ-overexpressing transgenic rice seedlings grown under normal and $\mathrm{NaCl}$-treated conditions, respectively, while 962 and 707 DEGs were downregulated (Fig. 6, Additional file 3: Data S1). Additionally, a four-way Venn diagram showed that a total of 922 DEGs were common to the shoots of OsERF106MZoverexpressing transgenic rice seedlings grown under both conditions, among which 486 and 436 DEGs were consistently up- and downregulated, respectively, under both conditions (Fig. 6, Additional file 4: Data S2). As expected, several abiotic stress-related genes such as OsABI5 (ABA-insensitive 5), OsMAIF1 (an F-box protein gene), and OsSRO1c (similar to RCD [radical-induced cell death] One 1c), were found among these commonly regulated DEGs. Accordingly, a qPCR assay was conducted

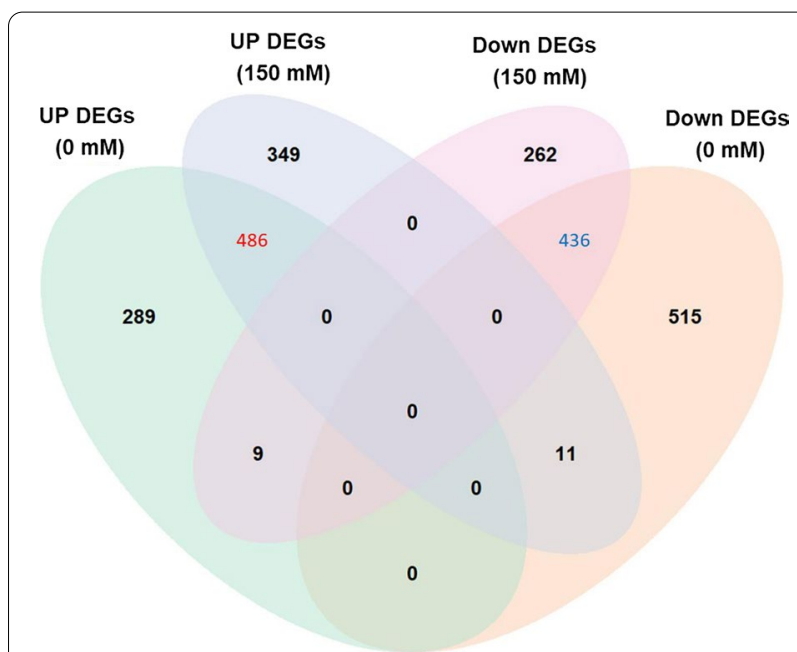

Fig. 6 Venn diagram analysis of the DEGs between Tainung 67 and OsERF106MZ-overexpressing rice shoots

to verify the microarray data. As shown in Fig. 7, the mRNA levels of OsABI5, OsHOX24 (rice homeodomainleucine zipper I subfamily member), OsMAIF1, and OsSRO1c were higher in OsERF106MZ-overexpressing shoots than in Tainung 67 shoots under both conditions, while the mRNA level of OsNACO06 was lower in OsERF106MZ-overexpressing shoots than in Tainung 67 shoots. The results obtained by qPCR were consistent with the microarray data. In addition, gene ontology analysis revealed that the major ontological categories of the commonly regulated DEGs were 'biological regulation' and 'cell wall organization or biogenesis' under the 'biological process' category and 'electron carrier activity' and 'transcription regulator activity' under the 'molecular function' category (Additional file 1: Figure S11). Incidentally, further promoter analysis indicated that 50 and 23 common up- and downregulated DEGs, respectively, contained at least one GCC box, an ERF-binding CRE, in their 1-kb promoter region, including OsMAIF1 (Additional file 4: Data S2).

\section{Discussion}

\section{Accurate Annotation of OsERF106}

Accurate annotation of gene structure is an initial step toward functional analysis of its roles in regulating plant growth, development, and responses to stress. Sometimes, it is not easy to accurately predict the coding region of a gene owing to the sequencing coverage and quality or an overlap of two individual genes in their coding regions. The AP2/ERF proteins comprise one of the largest TF families in plants. The AP2/ERF family is defined by containing one or two AP2/EREBP homology domains, which are responsible for sequence-specific 


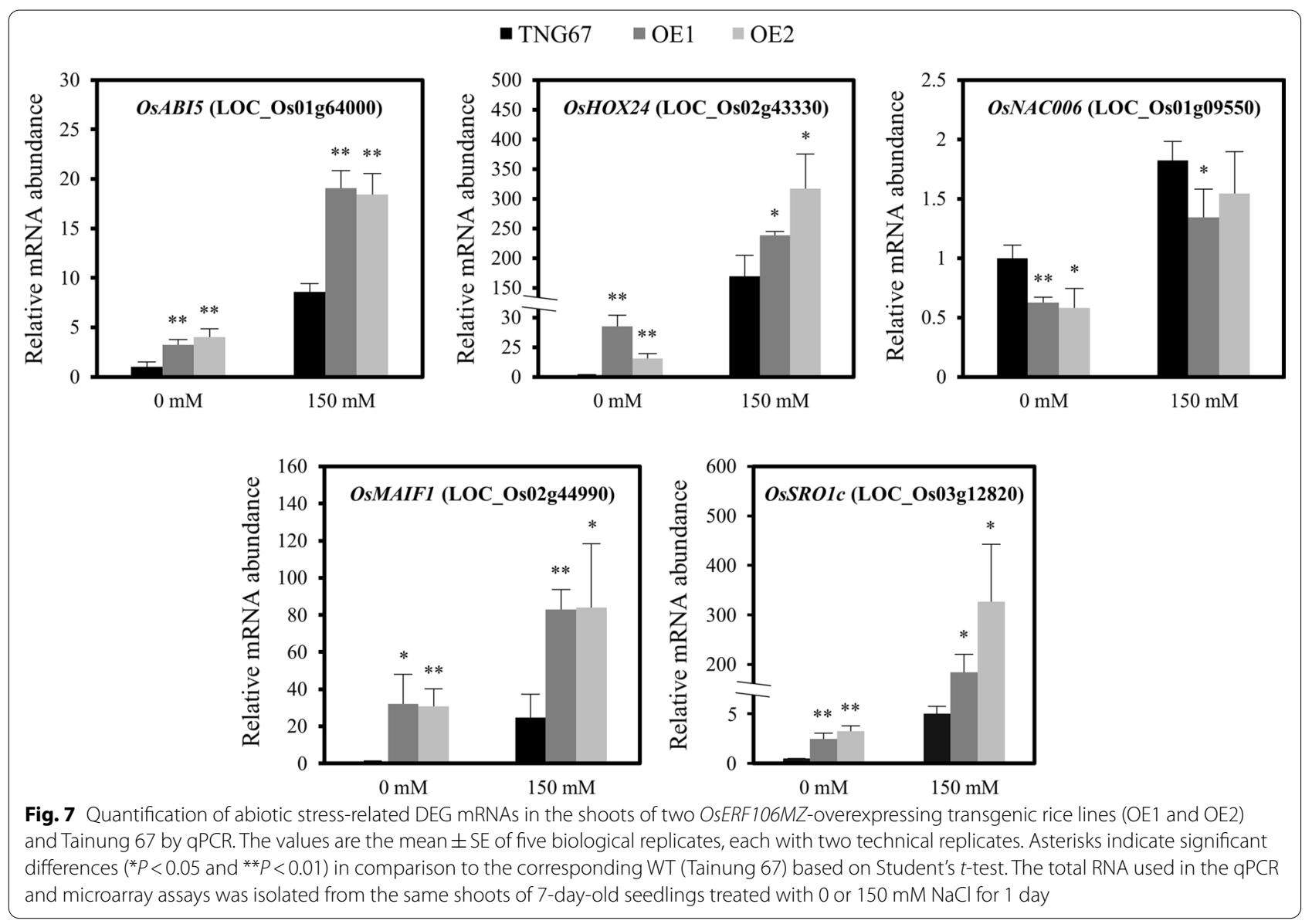

DNA binding (Ohme-Takagi and Shinshi 1995; Nakano et al. 2006). As noted by Nakano et al. (2006), the members of the ERF subfamily can be further subdivided into 8 (V-X, VI-like, and Xb-like) and 11 groups (V-XIV and VI-like) in Arabidopsis and japonica rice, respectively, according to their gene structure and conserved motifs (Nakano et al. 2006). Most of these groups are present in both Arabidopsis and japonica rice, indicating that the structural evolution and functional diversification of the plant ERF subfamily may predate monocot/dicot divergence. Additionally, it has been reported that most of the $A t E R F$ genes possess no introns, and only a few of these genes contain a single intron (Sakuma et al. 2002; Nakano et al. 2006). These findings imply that the structure and function of the OsERF gene should be similar to those of its homologs in Arabidopsis and rice. In the present study, several abiotic stress-responsive OsERF genes of unknown function were found by analyzing publicly available microarray datasets (GSE6901 and GSE14275). Among these genes, the expression of the OsERF106 gene was upregulated under salinity stress (Fig. 1a). However, the OsERF106 gene was differently annotated in the RAP-DB and MSU RGAP. Two (Locus tag: Os08t0537900-01 and Os08t0537900-02) and six (Locus tag: LOC_Os08g42550.1 to LOC_Os08g42550.6) putative OsERF106 transcript variants in the RAP-DB and MSU RGAP, respectively, were recorded (Additional file 1: Figure S2). Among these putative OsERF106 loci, Os08t0537900-01 as well as LOC_Os08g42550.2 to LOC_Os08g42550.6 contain six exons and five introns, while LOC_Os08g42550.1 consists of eight exons and seven introns. Notably, it has been reported that OsERF106 is highly homologous to OsERF105 (LOC_ Os05g36100) and OsERF107 (LOC_Os02g32140), which belong to the Xc subgroup of the rice ERF subfamily (Nakano et al. 2006). Additionally, as indicated on the MSU RGAP website, the OsERF105 (LOC_Os05g36100) and OsERF107 (LOC_Os02g32140) genes are orthologous to AtERF108/RAP2.6 (At1g43160) and AtERF113/ RAP2.6L (At5g13330), two members of the $\mathrm{X}$ group within the Arabidopsis ERF subfamily. However, almost all of the ERF genes in the Arabidopsis X group as well as the OsERF105 (LOC_Os05g36100) and OsERF107 (LOC_Os02g32140) genes are composed of two exons and one intron. Therefore, the gene structure of the Os08t0537900-01 and LOC_Os08g42550.s loci does 
not seem to conform to the conventional rules of $E R F$ genes possessing one intron at most and sharing high structural similarity with their homologs. Although the exon-intron organization of the Os08t0537900-02 locus conforms to these rules, its CDS is not consistent with the known characteristics of eukaryotic genes because it lacks an ATG start codon (Additional file 1: Figures S2 and S4).

Beyond their gene structure or the presence of an intact CDS, the proteins encoded by these putative OsERF106 loci are not easy to interpret. Among these putative OsERF106 loci, Os08t0537900-01 and LOC_Os08g42550.2 to LOC_Os08g42550.6 encode an $\mathrm{O}$-fucosyltransferase (O-FucT) that does not contain an AP2/EREBP domain, which is a defining feature of AP2/ ERF proteins (Additional file 1: Figures S2 and S3). The CDS of Os08t0537900-02 encodes an unknown protein that also lacks the AP2/EREBP domain, even though its CDS lacks an ATG-start codon (Additional file 1: Figures S2 and S4). LOC_Os08g42550.1 encodes an AP2/ EREBP domain-containing O-FucT-like protein; however, this protein is homologous to AtO-FucT13 and GmO-FucT13 and is not homologous to any AtAP2/ ERFs or GmAP2/ERFs, similar to the situation in the proteins encoded by Os08t0537900-01 and other LOC Os08g42550 loci (Additional file 1: Figures S2, S3, and S5). Notably, O-FucT is an enzymatic protein and is not a TF, and it is responsible for transferring $\mathrm{O}$-fucose from a donor substrate, such as guanosine diphosphate-fucose (GDP-fucose), to a protein (Ma et al. 2006; Tu et al. 2013). Therefore, a DBD, such as an AP2/EREBP domain, does not seem to be required for the enzymatic function of the O-FucT protein. In addition, qPCR analysis revealed that the RNA levels of LOC_Os08g42550 in Oserf106mz, a Tos17 mutant H0159 line harboring a Tos17 insertion within intron 7 of the LOC_Os08g42550.1 locus, were not significantly different from those in WT Hitomebore plants when the primer pair O-FucT-qF and O-FucTqR was used to amplify a common fragment of $72 \mathrm{bp}$ containing a sequence encoding part of O-FucT from LOC_Os08g42550.s (Additional file 1: Figure S9A and C). Based on these unusual annotation results, we speculated that O-FucT is encoded by an individual gene upstream of the OSERF106 gene and is not encoded by part of the OsERF106 gene. Accordingly, RACE-PCR using GSPs was performed to clarify the CDS of the OsERF106 gene. The RACE PCR-amplified OsERF106, designated OsERF106MZ, comprised two exons separated by an intron of $753 \mathrm{bp}$, and the encoded protein included an AP2/EREBP domain with conserved alanine and aspartic acid residues (Additional file 1: Figure S6). Additionally, OsERF106MZ is homologous to OsERF105 (LOC_Os05g36100), OsERF107 (LOC_Os02g32140), and their Arabidopsis homologs AtERF108/RAP2.6 (At1g43160) and AtERF113/RAP2.6L (At5g13330) but is not highly homologous to AtO-FucT13 and GmOFucT13 (Additional file 1: Figure S7). In fact, the CDS of OsERF106MZ is almost identical to the CDS of Os08t0537900-02, except for (1) an 11 bp insertion at the beginning of exon 2 in the OSERF106MZ gene, (2) a $31 \mathrm{bp}$ insertion at the end of exon 2 in the OsERF106MZ gene, and (3) a 3' UTR downstream of the TAA stop codon of the OsERF106MZ gene (Additional file 1: Figure S12). Notably, OsERF106MZ protein does not specifically localize to the nucleus, presumably due to the lack of a nuclear localization signal (NLS); likewise, both the OsERF105 (LOC_Os05g36100) and OsERF107 (LOC_ Os02g32140) proteins lack an NLS (Additional file 1: Figure S13).

\section{OsERF106MZ, a Member of the Rice ERF-X Group, Negatively Regulates the Salinity Stress Response by Disrupting ion Homeostasis and Modulating Stress-Responsive Gene Expression}

The AP2/ERF TF family plays an important role in regulating many aspects of plant growth and development (Licausi et al. 2013). Among the four AP2/ERF subfamilies, the role of CBF/DREB proteins in abiotic stresses has been extensively examined because several members of this subfamily, such as CBFs/DREB1s and DREB2s, play a major role in cold, drought, or salinity tolerance through the direct regulation of stress-responsive genes, including COR15a and COR78/RD29A (Lata and Prasad 2011; Mizoi et al. 2012; Xie et al. 2019). In addition to these members of the CBF/DREB subfamily, a growing number of members within the ERF subfamily, especially in the ERF-X group, have emerged as crucial regulators of various stress responses. In Arabidopsis and rice, there are 8 (AtERF108 to AtERF115) and 13 (OsERF98 to OsERF107, OsERF118, OsERF124, and OsERF125) genes belonging to the ERF-X group, respectively (Nakano et al. 2006). In Arabidopsis, overexpression of AtERF108/RAP2.6 (At1g43160) causes hypersensitivity to ABA and abiotic stresses during seed germination and early seedling development (Zhu et al. 2010). AtERF111/ABR1 (ABA repressor 1 [At5g64750]) acts as a transcriptional activator to regulate the wounding response (Bäumler et al. 2019). AtERF113/RAP2.6L (At5g13330) overexpression delays waterlogging-induced premature senescence, probably through an ABI1-mediated ABA signaling pathway (Liu et al. 2012a, b). Arabidopsis seedlings carrying a mutation in AtERF115 (At5g07310) are more sensitive to $75 \mathrm{mM}$ and $100 \mathrm{mM} \mathrm{NaCl}$ than WT seedlings (Krishnamurthy et al. 2017). Therefore, these studies have illustrated or implied the importance of plant ERF-X group members in regulating abiotic stress responses. Notably, 
more than half of the rice ERF-X group members are also abiotic stress-responsive genes. Among the 13 members of the rice ERF-X group, 9 genes are upregulated under different abiotic stress conditions, including OsERF106, which is specifically upregulated by salinity stress (for detail, see Mishra et al. 2013). In addition, almost all of these abiotic stress-responsive OsERF-X group genes are commonly expressed during both seed and reproductive developmental stages (Mishra et al. 2013). Although the expression profile of rice ERF-X group genes in response to abiotic stresses has been investigated in detail, their functional roles in regulating abiotic stress responses are still poorly understood. In this article, we examined the roles of OsERF106MZ, a salinity stress-responsive OsERF-Xc-type gene, in regulating the growth and salinity stress response of rice plants. As shown in Figs. 1 and $2 \mathrm{a}-\mathrm{d}$, histochemical GUS staining and qPCR assays confirmed that the expression of OsERF106MZ is enhanced by $\mathrm{NaCl}$ and is detectable in germinating seeds and developing flowers (a reproductive tissue), similar to the findings of Mishra et al. (2013). The overexpression of OsERF106MZ in rice can cause a severe retardation of shoot growth accompanied by relatively high levels of both MDA and ROS and a depression of CAT activity; however, no obvious differences in these morphological, physiological, and biochemical traits were found between the shoots of the Oserf $106 \mathrm{mz}$ mutant and its corresponding WT Hitomebore seedlings (Figs. 3 and 4). Further analysis revealed that OsERF106MZ-overexpressing transgenic rice seedlings accumulated excess $\mathrm{Na}^{+}$and $\mathrm{K}^{+}$ions in whole plants and shoots, respectively (Fig. 5a, Additional file 1: Figure S10B). Moreover, the expression of OsHKT1.3 was downregulated in OsERF106MZ-overexpressing shoots under both normal and $\mathrm{NaCl}$-treated conditions, while the expression of OsAKT1 was upregulated in transgenic shoots under $\mathrm{NaCl}$-treated conditions (Fig. 5b). The OsHKT1.s-encoded proteins are $\mathrm{Na}^{+}$-selective transporters, which are mostly located at the plasma membrane in xylem parenchyma cells (Hamamoto et al. 2015). OsHKT1.s proteins play a critical role in retrieving $\mathrm{Na}^{+}$from xylem sap, which prevents $\mathrm{Na}^{+}$overaccumulation in shoots. In addition, OsAKT1 encodes an inward $\mathrm{K}^{+}$channel that specifically localizes at the plasma membrane (Fuchs et al. 2005; Li et al. 2014). The expression of OsAKT1 is downregulated in both the shoots and roots of rice seedlings under salinity stress (Fuchs et al. 2005). However, the overexpression of OsAKT1 significantly increases the content of $\mathrm{K}^{+}$ in these tissues under osmotic/drought stress (Ahmad et al. 2016). Therefore, we speculated that the overaccumulation of $\mathrm{Na}^{+}$and $\mathrm{K}^{+}$ions in OsERF106MZ-overexpressing shoots may partially result from the interference of OsERF106MZ in the expression of OsHKT1.3 and
OsAKT1, which increases the toxicity of ions to cells and, thus, retards shoot growth in transgenic rice. In fact, a number of TFs have already been demonstrated to be involved in coordinately managing ion homeostasis and salinity stress responses. For example, OsbHLH035 mediates the expression of OsHKT1.3 and OsHKT1.5 in the aerial and terrestrial tissues of rice seedlings, respectively, which fine-tunes the response to salinity stress (Chen et al. 2018). OsSTAP1, a rice ERF-VIIa subgroup member, increases the expression of OsHKT8 and accordingly reduces the content of $\mathrm{Na}^{+}$ions in the shoots of rice plants, which contributes to the ability of rice plants to tolerate salinity stress (Wang et al. 2020). Thus, the function of OsERF106MZ seems to also be involved in the TF-mediated regulation of ion homeostasis in rice.

In addition to the regulation of ion homeostasis, the expression of several stress-responsive genes was altered in OsERF106MZ-overexpressing shoots under either normal or $\mathrm{NaCl}$-treated conditions. Among these genes, the expression of OsABI5, OsHOX24, OsMAIF1, and OsSRO1c was consistently upregulated, while the expression of OsNACO06 was consistently downregulated in OSERF106MZ-overexpressing shoots under both conditions (Fig. 7). OsABI5 encodes a nuclear-localized bZIP-type TF whose expression is induced by $\mathrm{ABA}$ and high salinity but downregulated by drought and cold (Zou et al. 2008). The overexpression of OsABI5 leads to an increase in sensitivity to salinity stress, while the repression of OsABI5 expression improves stress tolerance in transgenic rice. Additionally, rice transgenic lines overexpressing OsHOX24 exhibit extreme susceptibility to abiotic stresses at the seed germination stage and show severe retardation of seedling growth under salinity and desiccation stresses (Bhattacharjee et al. 2017). OsSRO1c, a direct target gene of SNAC1 (stress-responsive NAC 1), is involved in the oxidative stress response (You et al. 2013). The activity of enzymatic antioxidants, such as CAT, is significantly suppressed in OsSRO1c-overexpressing transgenic rice, and this transgenic rice is hypersensitive to oxidative stress. OsMAIF1 is a rice F-box domain gene, and its expression is rapidly induced by abiotic stresses (Yan et al. 2011). The overexpression of OsMAIF1 negatively regulates the abiotic stress tolerance but positively regulates the root growth of transgenic rice. Thus, we speculated that OsERF106MZ acts as a negative regulator of salinity stress via the disruption of ion homeostasis and the modulation of stress-responsive gene expression. Incidentally, despite excessive $\mathrm{NA}^{+}$ accumulation, OsERF106MZ-overexpressing transgenic seedlings do not show significant growth retardation of the roots. We suspected that the adverse effects of $\mathrm{Na}^{+}$excess on the root growth of transgenic rice 
overexpressing OsERF106MZ may be masked by the OsMAIF1-mediated promotion of root elongation.

\section{The OsERF106MZ-Mediated Negative Regulation of Salinity Stress Responses may Provide a Balance That Adjust Plant Responses to Salinity Stress}

In Arabidopsis, several CBF/DREB-IIIc-type TFs act as positive regulators of cold tolerance (for a review, see Xie et al. 2019). Notably, several CBF/DREB-IIatype TFs, such as AtERF011/DEAR1 (DREB and EAR motif protein 1 [At3g50260]) and AtERF006/RAP2.1 (At1g46768), are also upregulated during cold acclimation and serve as negative regulators of the cold stress response (Fowler and Thomashow 2002). AtERF011/ DEAR1 and AtERF006/RAP2.1 likely act upstream and downstream, respectively, of CBF/DREB-IIIc-type TFs (Tsutsui et al. 2009; Dong and Liu 2010). Overexpression of $A t E R F 011 / D E A R 1$ suppressed the cold-induced expression of $C B F S / D R E B s$ and resulted in drastically reduced freezing tolerance (Tsutsui et al. 2009). Similarly, Arabidopsis plants overexpressing AtERF006/ $R A P 2.1$ exhibited enhanced sensitivity to cold stress (Dong and Liu 2010). In fact, the TF-mediated negative regulation of abiotic stress responses is also found ubiquitously in crops. In Brassica napus, the earlyexpressed Group I DREBs switch on the CRT/DREmediated signaling pathway to increase plant tolerance to cold stress, whereas the late-expressed Group II DREBs competitively inhibited Group I DREB function to maintain a balance between plant growth and the cold stress response (Zhao et al. 2006). In the present study, rice seedlings overexpressing OsERF106MZ, a salinity-induced ERF-Xc-type TF, exhibited decreased tolerance to salinity stress with an increased $\mathrm{Na}^{+} / \mathrm{K}^{+}$ ratio in the shoots, which was quite similar to the findings in rice plants overexpressing OsERF922 (LOC_ Os01g54890), a salinity-induced ERF-IXa-type TF (Liu et al. 2012a, b). Therefore, the negative regulation of abiotic stress responses by abiotic stress-induced TF repressors in plants seems to provide a balance that minimizes the adverse effects of prolonged stress responses (Xie et al. 2019).

\section{Conclusions}

In the present study, we isolated a novel OsERF106, designated OsERF106MZ, by using RACE-PCR. The expression of OsERF106MZ is induced by salinity stress. The overexpression of OsERF106MZ leads to an overaccumulation of $\mathrm{Na}^{+}$and $\mathrm{K}^{+}$ions in the shoots of transgenic rice, resulting in a severe retardation of shoot growth. Moreover, the overexpression of OsERF106MZ interferes with the expression of OsHKT1.3, OsAKT1, and several stress-responsive genes under normal or $\mathrm{NaCl}$-treated conditions. All of the results suggest that OsERF106MZ negatively regulates salinity tolerance by disrupting ion homeostasis and modulating stress-responsive gene expression.

\section{Methods \\ Plant Materials, Growth Conditions, and Experimental Methods}

The Oserf106mz mutant (Tos17 line H0159) and its corresponding WT cultivar (Oryza sativa L. spp. japonica cv. Hitomebore) were obtained from the National Institute of Agrobiological Sciences (NIAS, Japan). Two independent OsERF106MZ-overexpressing transgenic rice lines (35Sp::OsERF106MZ [OE1] and 35Sp::OsERF106MZGFP [OE2]) were generated by Agrobacterium-mediated transformation. In all experiments, the seeds were treated with $1.5 \%(\mathrm{v} / \mathrm{v})$ commercial bleach for $30 \mathrm{~min}$, rinsed twice with sterile water for $30 \mathrm{~min}$ each time, and subsequently subjected to imbibition at $37{ }^{\circ} \mathrm{C}$ for 3 days in the dark. After imbibition, the germinating seeds were grown on a wire stand in a beaker at $28 / 24{ }^{\circ} \mathrm{C}$ (day/night) under long-day (16-h light/8-h dark) conditions with a light intensity of approximately $100 \mu \mathrm{E} \mathrm{s}^{-1} \mathrm{~m}^{2}$. The basal medium used in all of the experiments was half-strength Kimura B solution (Yoshida et al. 1976).

\section{Isolation of Rice OsERF106MZ Gene}

The full-length cDNA of OsERF106MZ gene was obtained from 5' and 3' cDNAs generated using a rapid amplification of cDNA ends (RACE) system (Clontech). Phylogenetic analysis revealed that OsERF106 (LOC_ Os08g42550) was highly homologous to OsERF105 (LOC_Os05g36100) and OsERF107 (LOC_Os02g32140) based on the sequence similarity of the AP2/EREBP domains (Nakano et al. 2006). Therefore, the genespecific primers (GSPs) used in the 5' and 3' RACE experiments were located downstream of the region encoding the AP2/EREBP domain of OsERF106 (LOC_ Os08g42550.1), the nucleotide sequence of which was highly divergent from those of OsERF105 and OsERF107 (Additional file 1: Figure S14). The primer positions and sequences are shown in Additional file 1: Figure S14 and Additional file 2: Table S1, respectively.

\section{RNA Extraction, cDNA Synthesis, and Quantitative PCR (qPCR)}

Total RNA was extracted from various tissues of rice using an RNeasy Plant Mini Kit (Qiagen) according to the manufacturer's instructions. To minimize genomic DNA contamination, up to $8 \mu \mathrm{g}$ of total RNA was treated after extraction with Turbo DNA-free ${ }^{\mathrm{TM}}$ DNase (Ambion) following the manufacturer's instructions, and $3 \mu \mathrm{g}$ of 
DNase-treated total RNA was then subjected to cDNA synthesis using the SuperScript ${ }^{\mathrm{TM}}$ III first-strand synthesis system (Invitrogen) according to the manufacturer's instructions. qPCR was carried out in an ABI 7500 system using the SYBR $^{\circledR}$ Green PCR Master Mix Kit (Applied Biosystems [ABI]). The initial amount of template cDNA in each amplification reaction was $10 \mu \mathrm{g}$. At least three independent biological replicates were performed for each experiment, and OsACTIN1 (LOC_ Os03g50885) was used as an internal control for qPCR normalization. The $2^{-\Delta \Delta C T}$ method was used to transform threshold cycle values $(\mathrm{Ct})$ into normalized relative abundance values of mRNA. The sequences of primers used for qPCR are presented in Additional file 2: Table S1.

\section{Transgene Constructs and Isolation of Transgenic Rice}

The full-length CDSs of OsERF106MZ and GFP were PCR-amplified with or without stop codons and cloned into the pGEM-T Easy vector (Promega). As shown in parentheses below, these fragments were subcloned into the binary pCAMBIA-1300 vector, in which their expression was driven by a CaMV $35 \mathrm{~S}$ promoter (35Sp::OsERF106MZ [OE1] and 35Sp::OsERF106MZGFP [OE2]). After the constructs were confirmed by sequencing, they were transformed into the rice cv. Tainung 67 background for subcellular OsERF106MZ localization and functional assays. Because the stable $\mathrm{T}_{0}$ rice transformants were chimeric and to avoid the undesirable side effects of multiple T-DNA insertions, $\mathrm{T}_{1}$ seeds were harvested from individual panicles of independent transformant lines that showed a 3:1 ratio of hygromycin resistance and sensitivity normalized to seed viability and were used to further screen for homozygous transgenic rice. Additionally, the $2.3-\mathrm{kb}$ OsERF106MZ promoter was cloned into pCAMBIA-1305.1 (OsERF106MZp::GUS), and the construct was transformed into the rice cv. Tainung 67 background for the investigation of the spatiotemporal pattern of OsERF106MZ expression. The spatiotemporal OsERF106MZ expression and subcellular OsERF106MZ localization assays were carried out as previously described (Chen et al. 2017).

\section{Measurement of MDA Content and Electrolyte Leakage}

For the MDA and electrolyte leakage assays, the seedlings were transferred to basal medium supplemented with 0 or $150 \mathrm{mM} \mathrm{NaCl}$ after growing for 7 days in beakers. Twenty-four hours later, the seedlings were used as initial materials for the measurement of MDA content or electrolyte leakage. The MDA content was determined following the method of Esterbauer and Cheeseman (1990). The aerial parts of the plant material were excised and vacuum dried overnight. Up to $0.1 \mathrm{~g}$ of the dried samples was added to $0.4 \mathrm{~mL}$ of $5 \%$ (w/v) trichloroacetic acid (TCA). After homogenization, the mixture was centrifuged at $10,000 \times g$ for $5 \mathrm{~min}$ at $20{ }^{\circ} \mathrm{C}$. Then, $0.1 \mathrm{~mL}$ of the supernatant was added to $0.4 \mathrm{~mL}$ of $0.5 \%(\mathrm{w} / \mathrm{v})$ thiobarbituric acid (TBA) dissolved in $20 \%(\mathrm{w} / \mathrm{v})$ TCA, followed by incubation for $30 \mathrm{~min}$ at $95^{\circ} \mathrm{C}$ in a water bath. After incubation, the mixture was removed from the water bath and centrifuged at $3000 \times g$ for $10 \mathrm{~min}$ at $4{ }^{\circ} \mathrm{C}$. The absorbance of the supernatant at 450,532 , and $600 \mathrm{~nm}$ was measured with a spectrometer. The MDA content was calculated as follows: MDA $\left(\mu \mathrm{mol} \mathrm{g}{ }^{-1}\right.$ dry weight $\left.[\mathrm{DW}]\right)=[6.452 \times(\mathrm{A} 532-\mathrm{A} 600)$ $-0.56 \times \mathrm{A} 450] \times 20 \div \mathrm{DW}(\mathrm{g})$. Electrolyte leakage was measured according to the method described by Bajji et al. (2002). The aerial parts of the plant material were washed twice with distilled water and then cut into 0.25to 0.5 -cm-long pieces. An approximately 0.05 -g sample of the leaf pieces was placed in a boiling tube filled with $50 \mathrm{~mL}$ of distilled water, which was then incubated in the dark for $24 \mathrm{~h}$ to allow the diffusion of electrolytes. Following incubation for $24 \mathrm{~h}$, the initial electrical conductivity (R1) in the solution was determined using a conductivity meter (model 3173R, JENCO, China). Thereafter, all leaf pieces were subjected to autoclaving at $121{ }^{\circ} \mathrm{C}$ under a pressure of 15 pounds per square inch for $20 \mathrm{~min}$ to release the electrolytes completely, and the electrical conductivity (R2) in the solution was measured after $1 \mathrm{~h}$ of equilibration at room temperature. The percentage of electrolyte leakage was calculated as follows: $(\mathrm{R} 1 / \mathrm{R} 2) \times 100 \%$.

\section{3,3'-Diaminobenzidine (DAB) and Nitro Blue Tetrazolium (NBT) Staining Assays}

For the DAB and NBT staining assays, the seedlings were transferred to basal medium supplemented with 0 or $150 \mathrm{mM} \mathrm{NaCl}$ after growing for 7 days in beakers. Twenty-four hours later, the 2nd leaf of each plant was excised and subjected to DAB staining or NBT staining. For DAB and NBT staining, leaves were incubated in a DAB $\left(0.1 \%\right.$ [w/v] DAB, $10 \mathrm{mM} \mathrm{Na}_{2} \mathrm{HPO}_{4}, 0.05 \%$ Tween 20 , $\mathrm{pH} 3.8)$ or NBT $\left(0.05 \%[\mathrm{w} / \mathrm{v}] \mathrm{NBT}, 50 \mathrm{mM} \mathrm{Na}_{2} \mathrm{HPO}_{4}\right.$, $0.05 \%$ Tween 20, $\mathrm{pH} 7.8$ ) solution overnight at $27{ }^{\circ} \mathrm{C}$ under light. After staining, the leaves were soaked in $95 \%$ ethanol overnight to remove chlorophyll.

\section{Catalase (CAT) Assay}

For the CAT assay, the seedlings were transferred to basal medium supplemented with 0 or $150 \mathrm{mM} \mathrm{NaCl}$ after growing for 7 days in beakers. Twenty-four hours later, the aerial parts of the plants were excised and subjected to the analysis of CAT activity. An approximately $0.07-\mathrm{g}$ sample of the aerial tissues was added to $1 \mathrm{~mL}$ of $50 \mathrm{mM}$ sodium phosphate buffer ( $\mathrm{pH}$ 6.8). After homogenization, the mixture was centrifuged at $12,000 \times g$ for $20 \mathrm{~min}$ 
at $4{ }^{\circ} \mathrm{C}$. After centrifugation, the supernatant was used as the source of enzymes. Then, $0.2 \mathrm{~mL}$ of the supernatant was added to $2.7 \mathrm{~mL}$ of $100 \mathrm{mM}$ sodium phosphate buffer ( $\mathrm{pH} \mathrm{7.0)}$ and $0.1 \mathrm{~mL}$ of $1 \mathrm{M} \mathrm{H}_{2} \mathrm{O}_{2}$. The decrease in the absorbance at $240 \mathrm{~nm}$ was recorded for $1 \mathrm{~min}$. CAT activity was calculated using an extinction coefficient of $40 \mathrm{mM}^{-1} \mathrm{~cm}^{-1}$ and was expressed as $\mathrm{M} \mathrm{H}_{2} \mathrm{O}_{2}$ oxidized minutes ${ }^{-1} \mathrm{mg}^{-1}$ protein (Scandalios et al. 1983).

\section{Measurement of lon Contents}

For the measurement of $\mathrm{Na}^{+}$and $\mathrm{K}^{+}$levels, the seedlings were transferred to basal medium supplemented with 0 or $150 \mathrm{mM} \mathrm{NaCl}$ after growing for 7 days in beakers. Ninety-six hours later, the aerial parts of the plants were excised, washed twice with ultrapure water, and subsequently vacuum dried overnight. The dried samples were digested using nitric and hydrochloric acids at a 4:1 ratio, boiled at $200{ }^{\circ} \mathrm{C}$ for $2 \mathrm{~h}$, and subsequently filtered after $1 \mathrm{~h}$ of equilibration at room temperature. The levels of $\mathrm{Na}^{+}$and $\mathrm{K}^{+}$in the resulting filtrates were determined using an inductively coupled plasma optical emission spectrometer (ICP-OES, PerkinElmer Optima 8000).

\section{Microarray and Data Analysis}

For the microarray experiment, the seedlings were transferred to basal medium supplemented with 0 or $150 \mathrm{mM}$ $\mathrm{NaCl}$ after growing for 7 days in beakers. Twenty-four hours later, the aerial parts of the plant were subjected to RNA extraction according to the method described above. The DNase-treated total RNA was amplified and labeled with cyanine 3 (Cy3) using a Low-Input Quick Amp Labeling Kit, One-Color (Agilent, USA) following the manufacturer's instructions. Labeled cRNA was fragmented by incubation at $60^{\circ} \mathrm{C}$ for $30 \mathrm{~min}$. After fragmentation, labeled cRNA was pooled and hybridized to the Agilent Rice Gene Expression $4 \times 44 \mathrm{~K}$ Microarray (Agilent Technologies) as described by the manufacturer. The array image was analyzed with Feature Extraction software version 10.7.1.1 (Agilent Technologies) using the default setting. All microarray data are available at the Gene Expression Omnibus (GEO, http://www.ncbi.nlm. nih.gov/geo) under the accession number GSE160238. To identify DEGs, the raw microarray data were analyzed with the R/Bioconductor Limma package (Ritchie et al. 2015). The uploaded data were background-corrected using the 'normexp' method and normalized using the 'quantile' method. Each up- or downregulated DEP exhibited a $\log _{2}$-fold change $>1$ or $<1$, respectively, with a $P$-value $<0.05$ based on the FDR-corrected Student's $t$-test. Each DEP-annotated gene is listed and described in the corresponding Additional file. Common DEG ontology graphical analysis was conducted using agriGO v2.0 online software (Tian et al. 2017).

\section{Abbreviations}

ABA: Abscisic acid; aa: Amino acid; AP2/EREBP: APETALA2/ethylene-responsive element binding protein; CAT: Catalase; CDS: Coding DNA sequence; CRE: cisRegulatory (or cis-acting) element; CRT/DRE: C-repeat/dehydration-responsive element; DAB: 3,3'-Diaminobenzidine; DBD: DNA-binding domain; GFP: Green fluorescent protein; GSPS: Gene-specific primers; GUS: $\beta$-Glucuronidase; ICPOES: Inductively coupled plasma-optical emission spectrometry; MDA: Malondialdehyde; MSU RGAP: Michigan State University Rice Genome Annotation Project Database; NBT: Nitro blue tetrazolium; O-FucT: O-Fucosyltransferase; ORF: Open reading frame; qPCR: Quantitative PCR; RACE-PCR: Rapid amplification of CDNA ends-PCR; RAP-DB: Rice Annotation Project Database; ROS: Reactive oxygen species; RT-PCR: Reverse transcription-PCR; TFs: Transcription factors; TNG67: Tainung 67 (Oryza sativa L. spp. japonica cv. Tainung 67); UTR: Untranslated region.

\section{Supplementary Information}

The online version contains supplementary material available at https://doi. org/10.1186/s12284-021-00525-5.

Additional file 1. Figure $\mathbf{S} 1$ The number of transcription factors in the AP2/ERF, bHLH, WRKY, bZIP, MYB, NAC, C2H2, and other families in Arabidopsis, maize, indica rice, and japonica rice. The data were retrieved from the PlantTFDB v5.0 website (http://planttfdb.cbi.pku.edu.cn/).

Figure S2 Structural comparison of putative OsERF106-encoding transcripts between the Rice Annotation Project Database (RAP-DB, http:// rapdb.dna.affrc.go.jp/) and the Michigan State University Rice Genome Annotation Project (MSU RGAP, http://rice.plantbiology.msu.edu/). The coding sequences of two Os08t0537900 transcripts (upper panel) and six Os08g42550 transcripts (lower panel) were documented on the RAP-DB and MSU RGAP websites, respectively. One typical AP2/EREBP domain (red bar) is present in the Os08g42550.1-encoded protein. The ScanProsite tool of ExPASy (http://www.expasy.org/) was used to retrieve the AP2/EREBP domain. Figure S3 Os08t0537900-01 and Os08g42550.2 to Os08g42550.6 encode an O-fucosyltransferase (O-FucT) without the AP2/ EREBP domain. The data were analyzed with NCBI SmartBLAST (http:// blast.ncbi.nlm.nih.gov/smartblast/). Figure S4 The coding sequence (CDS) of Os08t0537900-02 lacks an ATG-start codon. The image was taken from the Rice Annotation Project Database (RAP-DB, http://rapdb.dna.affrc.go. jp/). Figure S5 Os08g42550.1 encodes an AP2/EREBP domain-containing O-fucosyltransferase (O-FucT)-like protein, which is homologous to AtOFucT13 and GmO-FucT13 but not to any AtAP2/ERFs or GmAP2/ERFs. The data were analyzed with NCBI SmartBLAST (http://blast.ncbi.nlm.nih. gov/smartblast/). Figure S6 Gene structure and amino acid sequence alignment of OsERF106MZ (GenBank accession No. MZ561461). (A) Structure of OsERF106MZ. (B) Amino acid sequence alignment of OsERF105, OsERF106MZ, and OsERF107 together with their homologs, AtERF108 and AtERF113. The predicted AP2/EREBP domain is underlined in green. The conserved alanine 14 and aspartic acid 19 residues of the AP2/EREBP domain are indicated with red and blue asterisks, respectively. Figure S7 Phylogenetic analysis of OsERF105, OsERF106MZ, and OsERF107 together with AtERF108, AtERF113, AtO-FucT13, and GmO-FucT13 using the neighbor-joining method. Numbers next to the descendants indicate confidence values based on the bootstrap method. Figure S8 The subcellular localization of OsERF106MZ-GFP and GFP-OsERF106MZ in Oncidium 'Sweet Sugar' suspension cells. Figure S9 Characterization of a retrotransposon insertion Oserf106mz mutant line, H0159. (A) A schematic diagram of the retrotransposon insertion site (indicated with a red triangle) in Os08g42550.1 and the OSERF106MZ gene. (B) Identification of homozygous $\mathrm{H} 0159$ lines by genomic DNA genotyping. The Arabic numerals represent the individual rice plants within each genotype. (C) Quantification of Os08g42550.s mRNA levels in Hitomebore and Oserf106mz plants by qPCR. The values are the mean \pm SE of five biological replicates, each with two technical replicates. The positions of the primers used for genotyping 
(B) and $\mathrm{qPCR}(\mathrm{C})$ are indicated by green and blue arrows, respectively, in (A). The primer sequences are listed in Additional file 2: Table S1. The seedlings were grown on basal medium for 11 days and then subjected to genotyping and qPCR assays. Figure S10 MDA (left panel) and $\mathrm{Na}^{+}$(right panel) levels in the roots of Tainung 67 and OsERF106MZoverexpressing rice plants. Figure $\mathrm{S} 11 \mathrm{GO}$ analysis of the common DEGs according to biological processes and molecular functions. Figure S12 Comparison between the coding sequence of Os08t0537900-02 and the cDNA sequence of OsERF106MZ gene from both the TNG67 and Hitomebore (Hito) backgrounds. The 5' UTR, exon 1, exon 2, and 3' UTR within the CDNA sequence of OSERF $106 \mathrm{MZ}$ gene are underlined in green, red, blue, and purple, respectively. The ATG-start and TAA-stop codons of OsERF106MZ gene are indicated by yellow boxes. Figure S13 Nuclear localization signal (NLS) prediction in OsERF106MZ as well as its homologs OsERF105 and OsERF107. The data were analyzed with NLS Mapper (http://nls-mapper.iab.keio.ac.jp/). OsbHLH068 is a nuclear-localized protein that has been documented in a previous study (Chen et al. 2017) and is used as a positive control. Figure S14 Nucleotide sequence alignment of OsERF105 (Os05g36100), OsERF106 (Os08g42550.1), and OsERF107 (Os02g32140), three genes belonging to the rice ERF-Xc subgroup, from the region encoding the AP2/EREBP domain (underlined in green) to the translation stop site. The positions of the OSERF106 gene-specific primers (GSPs) used in $5^{\prime}$ and $3^{\prime}$ RACE experiments are underlined in red and blue, respectively.

Additional file 2. Table S1 Primers used in this study.

Additional file 3. Data S1 OsERF106-related DEGs.

Additional file 4. Data S2 Common DEGs.

\section{Acknowledgements}

We thank Ms. Mei-Jane Fang (Live-Cell Imaging Core Laboratory) and Miss Shu-Jen Chou (DNA Microarray Core Laboratory) from the Institute of Plant and Microbial Biology (IPMB) at Academia Sinica, Taipei, Taiwan, for operating the confocal microscope and performing the microarray hybridization, respectively. We are also grateful to Mr. Jiang-Tai Wu (IPMB, Academia Sinica) and Miss Lin-Yun Kuang (Transgenic Plant Core Facility, Academia Sinica) for assistance with the transient expression assays and providing Agrobacteriummediated stable transformation services, respectively.

\section{Authors' contributions}

$\mathrm{H}-\mathrm{CC}$ was responsible for designing and conducting the experiments, data assembly, and writing the manuscript. T-CC helped in conducting experiments and carried out the microarray analysis. T-YC offered great help in microarray data processing. M-HC and M-HL were responsible for propagating the plant materials. M-CC conceived the study and supervised the experiments. All authors read and approved the final manuscript.

\section{Funding}

This work was supported by the Ministry of Science and Technology, Taipei, Taiwan (Grant No. NSC-107-2313-B-002-030-MY3 to M.-C.C.).

\section{Availability of data and materials}

All data supporting the conclusions of this article are provided within the article and its additional files.

\section{Declarations}

Ethics approval and consent to participate

Not applicable.

\section{Consent for publication}

Not applicable.

\section{Competing interests}

The authors declare that they have no competing interests.

\section{Author details}

${ }^{1}$ Department of Agronomy, National Taiwan University, No. 1, Section 4, Roosevelt Road, Taipei, Taiwan, ROC. ${ }^{2}$ Crop Science Division, Taiwan Agricultural Research Institute, Taichung, Taiwan.

Received: 1 November 2020 Accepted: 12 September 2021

Published online: 20 September 2021

\section{References}

Ahmad I, Mian A, Maathuis FJ (2016) Overexpression of the rice AKT1 potassium channel affects potassium nutrition and rice drought tolerance. J Exp Bot 67:2689-2698

Bajji M, Kinet JM, Lutts S (2002) The use of the electrolyte leakage method for assessing cell membrane stability as a water stress tolerance test in durum wheat. Plant Growth Regul 36:61-70

Bäumler J, Riber W, Klecker M, Müller L, Dissmeyer N, Weig AR, Mustroph A (2019) AtERF\#111/ABR1 is a transcriptional activator involved in the wounding response. Plant J 100:969-990

Bhattacharjee A, Sharma R, Jain M (2017) Over-expression of OsHOX24 confers enhanced susceptibility to abiotic stresses in transgenic rice via modulating stress-responsive gene expression. Front Plant Sci 8:628

Chen HC, Hsieh-Feng V, Liao PC, Cheng WH, Liu LY, Yang YW, Lai MH, Chang MC (2017) The function of OsbHLH068 is partially redundant with its homolog, AtbHLH112, in the regulation of the salt stress response but has opposite functions to control flowering in Arabidopsis. Plant Mol Biol 94:531-548

Chen HC, Cheng WH, Hong CY, Chang YS, Chang MC (2018) The transcription factor OsbHLH035 mediates seed germination and enables seedling recovery from salt stress through $A B A$-dependent and $A B A$-independent pathways, respectively. Rice $(n Y)$ 11:50

Chinnusamy V, Ohta M, Kanrar S, Lee BH, Hong X, Agarwal M, Zhu JK (2003) ICE1: a regulator of cold-induced transcriptome and freezing tolerance in Arabidopsis. Genes Dev 17:1043-1054

Deinlein U, Stephan AB, Horie T, Luo W, Xu G, Schroeder JI (2014) Plant salttolerance mechanisms. Trends Plant Sci 19:371-379

Dong CJ, Liu JY (2010) The Arabidopsis EAR-motif-containing protein RAP2.1 functions as an active transcriptional repressor to keep stress responses under tight control. BMC Plant Biol 10:47

Esterbauer H, Cheeseman KH (1990) Determination of aldehydic lipid peroxidation products: malonaldehyde and 4-hydroxynonenal. Methods Enzymol 186:407-421

Fowler S, Thomashow MF (2002) Arabidopsis transcriptome profiling indicates that multiple regulatory pathways are activated during cold acclimation in addition to the CBF cold response pathway. Plant Cell 14:1675-1690

Fuchs I, Stölzle S, Ivashikina N, Hedrich R (2005) Rice K+ uptake channel OsAKT1 is sensitive to salt stress. Planta 221:212-221

Hamamoto S, Horie T, Hauser F, Deinlein U, Schroeder Jl, Uozumi N (2015) HKT transporters mediate salt stress resistance in plants: from structure and function to the field. Curr Opin Biotechnol 32:113-120

Hong Y, Zhang H, Huang L, Li D, Song F (2016) Overexpression of a stressresponsive NAC transcription factor gene ONAC022 improves drought and salt tolerance in rice. Front Plant Sci 7:4

Isayenkov SV, Maathuis FJM (2019) Plant salinity stress: many unanswered questions remain. Front Plant Sci 10:80

Jiang C, lu B, Singh J (1996) Requirement of a CCGAC cis-acting element for cold induction of the BN115 gene from winter Brassica napus. Plant Mol Biol 30:679-684

Krishnamurthy P, Mohanty B, Wijaya E, Lee DY, Lim TM, Lin Q, Xu J, Loh CS, Kumar PP (2017) Transcriptomics analysis of salt stress tolerance in the roots of the mangrove Avicennia officinalis. Sci Rep 7:10031

Kumar K, Kumar M, Kim SR, Ryu H, Cho YG (2013) Insights into genomics of salt stress response in rice. Rice $(n Y)$ 6:27

Lata C, Prasad M (2011) Role of DREBs in regulation of abiotic stress responses in plants. J Exp Bot 62:4731-4748

Li J, Long Y, Qi GN, Li J, Xu ZJ, Wu WH, Wang Y (2014) The Os-AKT1 channel is critical for $\mathrm{K}+$ uptake in rice roots and is modulated by the rice CBL1CIPK23 complex. Plant Cell 26:3387-3402 
Licausi F, Ohme-Takagi M, Perata P (2013) APETALA2/Ethylene Responsive Factor (AP2/ERF) transcription factors: mediators of stress responses and developmental programs. New Phytol 199:639-649

Liu D, Chen X, Liu J, Ye J, Guo Z (2012a) The rice ERF transcription factor OsERF922 negatively regulates resistance to Magnaporthe oryzae and salt tolerance. J Exp Bot 63:3899-3911

Liu P, Sun F, Gao R, Dong H (2012b) RAP2.6L overexpression delays waterlogging induced premature senescence by increasing stomatal closure more than antioxidant enzyme activity. Plant Mol Biol 79:609-622

Liu Y, Ji X, Nie X, Qu M, Zheng L, Tan Z, Zhao H, Huo L, Liu S, Zhang B, Wang $Y$ (2015) Arabidopsis AtbHLH112 regulates the expression of genes involved in abiotic stress tolerance by binding to their E-box and GCGbox motifs. New Phytol 207:692-709

Liu J, Shi Y, Yang S (2018) Insights into the regulation of C-repeat binding factors in plant cold signaling. J Integr Plant Biol 60:780-795

Ma B, Simala-Grant JL, Taylor DE (2006) Fucosylation in prokaryotes and eukaryotes. Glycobiology 16:158R-184R

Maas EV, Hoffman GJ (1997) Crop salt tolerance. ASCE J Irrig Drain Div 103:115-134

Mishra M, Kanwar P, Singh A, Pandey A, Kapoor S, Pandey GK (2013) Plant omics: genome-wide analysis of ABA repressor1 (ABR1) related genes in rice during abiotic stress and development. OMICS 17:439-450

Mitchell PJ, Tjian R (1989) Transcriptional regulation in mammalian cells by sequence-specific DNA binding proteins. Science 245:371-378

Mizoi J, Shinozaki K, Yamaguchi-Shinozaki K (2012) AP2/ERF family transcription factors in plant abiotic stress responses. Biochim Biophys Acta 1819:86-96

Nakano T, Suzuki K, Fujimura T, Shinshi H (2006) Genome-wide analysis of the ERF gene family in Arabidopsis and rice. Plant Physiol 140:411-432

Ohme-Takagi M, Shinshi H (1995) Ethylene-inducible DNA binding proteins that interact with an ethylene-responsive element. Plant Cell 7:173-182

Ptashne M, Gann A (1997) Transcriptional activation by recruitment. Nature 386:569-577

Ritchie ME, Phipson B, Wu D, Hu Y, Law CW, Shi W, Smyth GK (2015) Limma powers differential expression analyses for RNA-sequencing and microarray studies. Nucleic Acids Res 43:e47

Sakuma Y, Liu Q, Dubouzet JG, Abe H, Shinozaki K, Yamaguchi-Shinozaki K (2002) DNA-binding specificity of the ERF/AP2 domain of Arabidopsis DREBs, transcription factors involved in dehydration- and cold-inducible gene expression. Biochem Biophys Res Commun 290:998-1009

Scandalios JG, Tsaftaris AS, Chandlee JM, Skadsen RW (1983) Expression of the developmentally regulated catalase (Cat) genes in maize. Dev Genet 4:281-293

Tian T, Liu Y, Yan H, You Q, Yi X, Du Z, Xu W, Su Z (2017) agriGO v2.0: a GO analysis toolkit for the agricultural community, 2017 update. Nucleic Acids Res 45:W122-W129
Tsutsui T, Kato W, Asada Y, Sako K, Sato T, Sonoda Y, Kidokoro S, YamaguchiShinozaki K, Tamaoki M, Arakawa K, Ichikawa T, Nakazawa M, Seki M, Shinozaki K, Matsui M, Ikeda A, Yamaguchi J (2009) DEAR1, a transcriptional repressor of DREB protein that mediates plant defense and freezing stress responses in Arabidopsis. J Plant Res 122:633-643

Tu Z, Lin YN, Lin CH (2013) Development of fucosyltransferase and fucosidase inhibitors. Chem Soc Rev 42:4459-4475

United State Department of Agriculture (2016) Research databases. Bibliography on salt tolerance. George E. Brown Jr. Salinity Lab., US Dep. Agric., Agric. Res. Serv., Riverside, CA

Wang Y, Wang J, Zhao X, Yang S, Huang L, Du F, Li Z, Zhao X, Fu B, Wang W (2020) Overexpression of the transcription factor gene OsSTAP1 increases salt tolerance in rice. Rice ( $\mathrm{n} Y$ ) 13:50

Xie Z, Nolan TM, Jiang H, Yin Y (2019) AP2/ERF Transcription factor regulatory networks in hormone and abiotic stress responses in arabidopsis. Front Plant Sci 10:228

Yan YS, Chen XY, Yang K, Sun ZX, Fu YP, Zhang YM, Fang RX (2011) Overexpression of an F-box protein gene reduces abiotic stress tolerance and promotes root growth in rice. Mol Plant 4:190-197

Yoshida S, Forno DA, Cock JH, Gomez KA (1976) Routine procedure for growing rice plants in culture solution. In: Laboratory manual for physiological studies of rice. International Rice Research Institute, Manila, Philippines

You J, Zong W, Li X, Ning J, Hu H, Li X, Xiao J, Xiong L (2013) The SNAC1targeted gene OsSRO1c modulates stomatal closure and oxidative stress tolerance by regulating hydrogen peroxide in rice. J Exp Bot 64:569-583

Zhao TJ, Sun S, Liu Y, Liu JM, Liu Q, Yan YB, Zhou HM (2006) Regulating the drought-responsive element (DRE)-mediated signaling pathway by synergic functions of trans-active and trans-inactive DRE binding factors in Brassica napus. J Biol Chem 281:10752-10759

Zhao C, Zhang Z, Xie S, Si T, Li Y, Zhu JK (2016) Mutational evidence for the critical role of CBF transcription factors in cold acclimation in Arabidopsis. Plant Physiol 171:2744-2759

Zhu Q, Zhang J, Gao X, Tong J, Xiao L, Li W, Zhang H (2010) The Arabidopsis AP2/ERF transcription factor RAP2.6 participates in ABA, salt and osmotic stress responses. Gene 457:1-12

Zou M, Guan Y, Ren H, Zhang F, Chen F (2008) A bZIP transcription factor, OsABI5, is involved in rice fertility and stress tolerance. Plant Mol Biol $66: 675-683$

\section{Publisher's Note}

Springer Nature remains neutral with regard to jurisdictional claims in published maps and institutional affiliations.

\section{Submit your manuscript to a SpringerOpen ${ }^{\circ}$ journal and benefit from:}

- Convenient online submission

- Rigorous peer review

- Open access: articles freely available online

- High visibility within the field

- Retaining the copyright to your article

Submit your next manuscript at springeropen.com 